\title{
A Lagrangian Approach for Computational Acoustics with Meshfree Method
}

\author{
Yong Ou Zhang ${ }^{1,2}$, Stefan G. Llewellyn Smith ${ }^{2}$, Tao Zhang ${ }^{1, *}$, Tian Yun $\mathrm{Li}^{1}$ \\ ${ }^{1}$ School of Naval Architecture and Ocean Engineering, Huazhong University of Science and \\ Technology, Wuhan 430074, China; zhangyo1989@gmail.com (Y. O. Zhang),sgls@ucsd.edu \\ (S. G. Llewellyn Smith), ltyz801@hust.edu.cn (T. Y. Li) \\ ${ }^{2}$ Department of Mechanical and Aerospace Engineering, University of California, San Diego, La \\ Jolla, CA 92093, USA \\ * Correspondence: zhangt7666@hust.edu.cn; Tel: +8613995559242
}

\begin{abstract}
Although Eulerian approaches are standard in computational acoustics, they are less effective for certain classes of problems like bubble acoustics and combustion noise. A different approach for solving acoustic problems is to compute with individual particles following particle motion. In this paper, a Lagrangian approach to model sound propagation in moving fluid is presented and implemented numerically, using three meshfree methods to solve the Lagrangian acoustic perturbation equations (LAPE) in the time domain. The LAPE split the fluid dynamic equations into a set of hydrodynamic equations for the motion of fluid particles and perturbation equations for the acoustic quantities corresponding to each fluid particle. Then, three meshfree methods, the smoothed particle hydrodynamics (SPH) method, the corrective smoothed particle (CSP) method, and the generalized finite difference (GFD) method, are introduced to solve the LAPE and the linearized LAPE (LLAPE). The SPH and CSP methods are widely used meshfree methods, while the GFD method based on the Taylor series expansion can be easily extended to higher orders. Applications to modeling sound propagation in steady or unsteady fluids in motion are outlined, treating a number of different cases in one and two space dimensions. A comparison of the LAPE and the LLAPE using the three meshfree methods is also presented. The Lagrangian approach shows good agreement with exact solutions. The comparison indicates that the CSP and GFD method exhibit convergence in cases with different background flow. The GFD method is more accurate, while the CSP method can handle higher Courant numbers.
\end{abstract}

Keywords: Lagrangian approach; Lagrangian acoustic perturbation equations; computational acoustics; meshfree method; smoothed particle hydrodynamics; generalized finite difference method 


\section{Introduction}

Computational acoustics is widely used in industrial applications to model devices such as fans, automobile manufactures, underwater vehicles, room acoustics, and so on. Many different models have been proposed and used in these applications, such as direct numerical methods [1], the linearized Euler equations (LEE) [2], acoustic analogy methods (Lighthill's acoustic analogy theory [3], Ffowcs-Williams and Hawkings (FW-H) method [4], etc...), and hydrodynamic/acoustic splitting methods (expansion around an incompressible flow (EIF) model $[5,6]$, acoustic perturbation equations (APE) [7, 8], perturbed compressible equations (PCE) $[9,10]$, etc...).

Eulerian approaches have mainly been used to implement numerical approximations to these models, and different numerical methods include the finite difference method (FDM) [11], the finite element method (FEM) [12], the boundary element method (BEM) [13], and other modified or coupled methods [14-16]. These methods have shown their power in solving various acoustic problems so far. However, they are still not ideal for solving certain acoustic problems, such as transient acoustic problems with free surfaces, complex material interfaces, inhomogeneous media, moving or deformable boundaries, and multiphase systems. Some specific examples are bubble acoustics [17], combustion noise [18], sound propagation in multiphase flows [19], etc...

Compared to the Eulerian approach which solves for quantities at fixed locations in space, the Lagrangian approach uses individual particles which move through both space and time, and have their own physical properties, such as density, velocity, and pressure, to represent the dynamically evolving fluid flow. The flow is described by recording the time history of each fluid particle. A comparison of two approaches is outlined in Fig. 1.

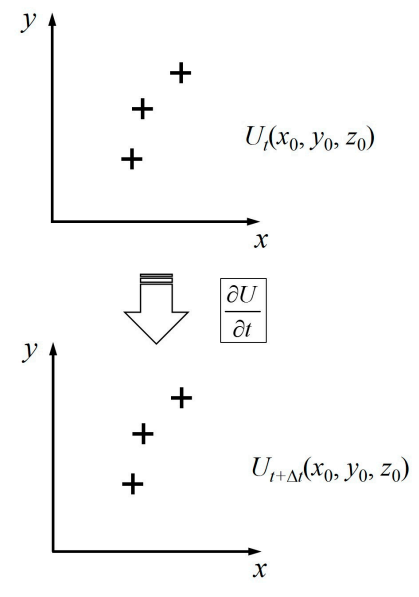

Eulerian Approach

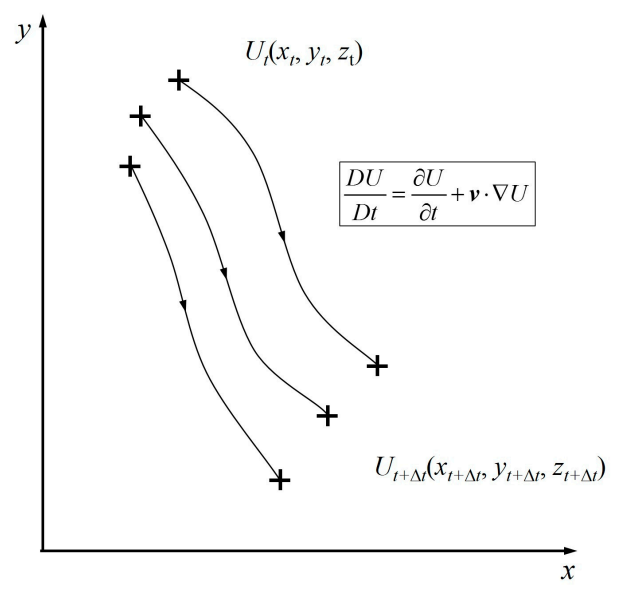

Lagrangian Approach

Fig. 1 Eulerian and Lagrangian approaches in numerical computation. ( $U$ stands for computational variables, $t$ stands for time, and + are computational points)

It should be noted that even for a Lagrangian approach, Lagrangian quantities are 
usually represented in an Eulerian framework, since equations written in the Lagrangian description are very awkward for most purposes, and are seldom used [20]. A comparison between Eulerian and Lagrangian descriptions can also be found in [20]. Consequently, a Lagrangian approach with Lagrangian quantities represented by the Eulerian description is an alternative way to deal with fluid dynamics problems or acoustic problems. The application of Lagrangian approaches in CFD has shown the advantages of such methods in solving problems with multiphase systems [21-23].

However, in the field of computational acoustics, there have been almost no applications of Lagrangian approaches. It is possible to solve the Navier-Stokes (N-S) equations directly over all spatial and temporal scales in a Lagrangian approach $[24,25]$, which can be viewed as a Lagrangian direct numerical simulation (DNS), but this Lagrangian DNS is as costly as an Eulerian DNS.

Moreover, it is worth noting that a partial Lagrangian method, which is also a hydrodynamic/acoustic splitting method, has been obtained previously: this partial Lagrangian method, which considers fluid flow with Eulerian quantities and represents acoustic variables with a Lagrangian description, had been used in Galbrun's equations [26, 27] for aeroacoustics. The perturbation of the particle position due to sound waves is the only Lagrangian variable used in this partial Lagrangian method. The flow field is computed based on fixed grids in this approach, and hence the advantages of the Lagrangian approach mentioned before cannot be used in the fluid flow computation.

The purpose of our work is to devise a Lagrangian approach for computational acoustics, which computes the fluid particle motion and the sound waves based on the acoustic perturbation assumption $[8,9]$. In this way, this approach can consider sound generation and propagation with complex background flow or multiple media, and can be more efficient than the Lagrangian DNS method by ignoring scale disparities since the flow and acoustics are treated separately $[10,28]$ as in hydrodynamic/acoustic splitting methods. In addition, we choose meshfree method, instead of traditional mesh based methods to realize the computation numerically, because dependent variables correspond fluid particles, which can move with the fluid, instead of fixed points.

Meshfree methods has been used to solve the acoustic wave equation in quiescent media in the time domain or the Helmholtz equation in the frequency domain, as in the multiplescale reproducing kernel particle method (RKPM) [29], the element-free Galerkin method (EFGM) [30], the method of fundamental solutions (MFS) [31], etc...[32, 33]. When using a meshfree method, the Lagrangian approach proposed in this paper not only has the advantages of the Lagrangian property mentioned before, but also has the benefits of the meshfree method itself, such as avoiding mesh generation. 
In this paper, we have chosen three different meshfree methods to implement and validate the Lagrangian acoustic perturbation equations (LAPE): smoothed particle hydrodynamic (SPH), the corrective smoothed particle method (CSP), and the generalized finite difference (GFD) method, a meshfree method derived from traditional finite difference methods. These three methods are representative of meshfree methods in general.

The SPH method is a Lagrangian meshfree method first pioneered independently by Lucy [34] and Gingold and Monaghan [35] to solve astrophysical problems in 1977. It has been widely used in different fields as outlined in recent reviews [21-23, 36]. Details about the standard SPH computation can be found in Liu and Liu's book [37], and the CSP method can be found in $[38,39]$. To date, this Lagrangian method has shown its advantages in fluid dynamics simulations, and the Lagrangian particle tracking method has been widely used in modelling bubble motions [40], free surface [41], dust [42], and other problems. In the preliminary work, we have given a compact discussion concerns the numerical performance of the SPH method in solving acoustic wave equations in quiescent media [43-45].

The GFD method, also called the meshless finite difference method, was first discussed for fully arbitrary meshes by Jensen [46] in 1972. Perrone and Kao [47] also contributed to the development of this method at that time. Subsequently, a variation of the GFD method using the moving least squares approximation was proposed by Lizska and Orkisz [48]. Recently, Benito, Urena and Gavete gave a discussion about the influence of several factors in the GFD method, and a comparison between the GFD method and the EFGM in solving Laplace equation is also presented in [49-51]. The GFD method shows more accurate than the EFGM. In addition, the GFD method was applied to solve acoustic wave equations in quiescent media recently [52].

The present paper is organized as follows. In section 2, the LAPE are obtained. In section 3, the SPH and CSP formulations for simulating acoustic waves in a moving flow is given based on the LAPE. In section 4, the GFD formulation for computing LAPE is shown. Several test cases are given in order to validate the numerical method in section 4 , while section 5 summarizes the results of this work.

\section{Lagrangian acoustic perturbation equations}

The fundamental assumptions and equations are presented first, and then the LAPE for computational acoustics are obtained.

\subsection{Fundamental assumptions and equations}

The basic idea of the hydrodynamic/acoustic splitting method is to split the background flow and acoustic perturbation based on differences in amplitude. Details of this approach 
can be found in $[8,9]$. Following this idea, consider a fluid particle which has physical properties:

$$
\begin{gathered}
P=p_{0}+\delta p,|\delta p|<<p_{0}, \\
\rho=\rho_{0}+\delta \rho,|\delta \rho|<<\rho_{0}, \\
\boldsymbol{v}=v_{0}+\delta \boldsymbol{v},|\delta \boldsymbol{v}|<<v_{0}, \\
S=S_{0}+\delta S,|\delta S|<<S_{0},
\end{gathered}
$$

where $P, \rho, v$, and $S$ are the pressure, density, velocity, and entropy of the fluid particle respectively; $p_{0}, \rho_{0}, v_{0}$, and $S_{0}$ are the components in the absence of sound, while $\delta p$, $\delta \rho, \delta v$, and $\delta S$ are the perturbations of corresponding parameters due to acoustic waves.

All these variables are supposed to be the physical properties of a fluid particle which can move through both space and time, rather than at a fixed position. Nevertheless, as mentioned in the introduction, all these parameters are represented in Eulerian coordinates.

The Lagrangian form of the fluid dynamics equations is given in Chapter 4 of Liu and Liu's book [37]. The fluid dynamics equations including gravity can be written as: the continuity equation

$$
\frac{D \rho}{D t}=-\rho \nabla \cdot v
$$

the momentum equation

$$
\frac{D \boldsymbol{v}}{D t}=-\frac{1}{\rho} \nabla P+\boldsymbol{g},
$$

the equation of state

$$
P=P(\rho, S),
$$

where the material or Lagrangian derivative is written as

$$
\frac{D}{D t}=\frac{\partial}{\partial t}+\boldsymbol{v} \cdot \nabla
$$

Now we consider the sound waves in an inhomogeneous medium without the effects of viscosity. Sound propagation can be modelled as an adiabatic process, and viscosity, diffusion and thermal conductivity can be neglected. Let $c$ be the sound velocity; then the equation of state, according to Chapter 4 in Brekhovskikh and Godin's book [8], becomes

$$
\frac{D P}{D t}=c^{2} \frac{D \rho}{D t}
$$

where 


$$
c=c(\rho, S)=\sqrt{\left(\frac{\partial P}{\partial \rho}\right)_{S}} .
$$

For adiabatic processes we have

$$
\frac{D S}{D t}=0
$$

Eq. (5), Eq. (6), and Eqs. (9)-(10) are a closed system for equations of fluid dynamics. For an ideal gas, Eq. (10) can be replaced by

$$
c^{2}=\frac{\gamma P}{\rho}
$$

where $\gamma$ is the ratio of specific heats ( $\gamma=1.4$ for air).

\subsection{Equations for background fluid motion}

Now we consider the flow without sound waves. The usual hydrodynamic equations include gravity can be written as

$$
\begin{gathered}
\frac{D_{0} \rho_{0}}{D t}=-\rho_{0} \nabla \cdot \boldsymbol{v}_{0}, \\
\frac{D_{0} \boldsymbol{v}_{0}}{D t}=-\frac{1}{\rho_{0}} \nabla p_{0}+\boldsymbol{g}, \\
p_{0}=p_{0}\left(\rho_{0}, S_{0}\right),
\end{gathered}
$$

where the material derivative is

$$
\frac{D_{0}}{D t}=\frac{\partial}{\partial t}+v_{0} \cdot \nabla
$$

For an adiabatic process neglecting viscosity, diffusion, and thermal conductivity, we have the equation of state

$$
\frac{D_{0} p_{0}}{D t}=c_{0}^{2} \frac{D_{0} \rho_{0}}{D t}
$$

where

$$
c_{0}=c_{0}\left(\rho_{0}, S_{0}\right)=\sqrt{\left(\frac{\partial p_{0}}{\partial \rho_{0}}\right)_{S_{0}}} .
$$

For an adiabatic process we have

$$
\frac{D_{0} S_{0}}{D t}=0
$$

where $c_{0}$ is the sound speed not influenced by the acoustic wave, with $c^{2}=c_{0}^{2}+\delta c^{2}$.

For an ideal gas, Eq. (18) can be replaced by 


$$
c_{0}^{2}=\frac{\gamma p_{0}}{\rho_{0}}
$$

\subsection{Lagrangian acoustic perturbation equations}

Considering acoustic perturbations generated by sound, and substituting Eqs. (1)-(3) into the continuity equation (Eq. (5)), the momentum equations (Eq. (6)), and the equation of state (Eq. (9)) leads to

$$
\begin{gathered}
\frac{D\left(\rho_{0}+\delta \rho\right)}{D t}=-\left(\rho_{0}+\delta \rho\right) \nabla \cdot\left(\boldsymbol{v}_{0}+\delta \boldsymbol{v}\right), \\
\frac{D\left(\boldsymbol{v}_{0}+\delta \boldsymbol{v}\right)}{D t}=-\frac{1}{\left(\rho_{0}+\delta \rho\right)} \nabla\left(p_{0}+\delta p\right)+\boldsymbol{g}, \\
\frac{D\left(p_{0}+\delta p\right)}{D t}=\left(c_{0}^{2}+\delta c^{2}\right) \frac{D\left(\rho_{0}+\delta \rho\right)}{D t} .
\end{gathered}
$$

Rearranging these equations using the hydrodynamic equations (Eqs. (13), (14), and (17)), we obtain the LAPE as

$$
\begin{gathered}
\frac{D_{0} \delta \rho}{D t}=-\rho_{0} \nabla \cdot \delta \boldsymbol{v}-\delta \rho \nabla \cdot\left(\boldsymbol{v}_{0}+\delta \boldsymbol{v}\right)-(\delta \boldsymbol{v} \cdot \nabla)\left(\rho_{0}+\delta \rho\right) \\
\frac{D_{0} \delta \boldsymbol{v}}{D t}=-\frac{1}{\rho_{0}} \nabla \delta p-(\delta \boldsymbol{v} \cdot \nabla)\left(\boldsymbol{v}_{0}+\delta \boldsymbol{v}\right)+\frac{\delta \rho}{\rho_{0}\left(\rho_{0}+\delta \rho\right)} \nabla\left(p_{0}+\delta p\right) \\
\frac{D_{0} \delta p}{D t}-c_{0}^{2} \frac{D_{0} \delta \rho}{D t}=-\delta \boldsymbol{v} \nabla\left(p_{0}+\delta p\right)+c_{0}^{2}(\delta \boldsymbol{v} \cdot \nabla)\left(\rho_{0}+\delta \rho\right) \\
+\delta c^{2}\left[\frac{D_{0} \rho_{0}}{D t}+\frac{D_{0} \delta \rho}{D t}+(\delta \boldsymbol{v} \cdot \nabla)\left(\rho_{0}+\delta \rho\right)\right]
\end{gathered}
$$

where the material derivative used is Eq. (16). These equations are exact.

Ignoring the second-order terms, leads to linear equations for sound waves. In order to take into account sound sources, two terms are added to the right hand side of equations, and the linearized LAPE (LLAPE) are

$$
\begin{gathered}
\frac{D_{0} \delta \rho}{D t}=-\rho_{0} \nabla \cdot \delta \boldsymbol{v}-\delta \rho \nabla \cdot v_{0}-(\delta \boldsymbol{v} \cdot \nabla) \rho_{0}+\delta \rho a \\
\frac{D_{0} \delta \boldsymbol{v}}{D t}=-\frac{1}{\rho_{0}} \nabla \delta p-\delta \boldsymbol{v} \nabla \cdot v_{0}+\frac{\delta \rho}{\left(\rho_{0}\right)^{2}} \nabla p_{0}+\frac{\delta \boldsymbol{f}}{\rho_{0}} \\
\frac{D_{0} \delta p}{D t}-c_{0}^{2} \frac{D_{0} \delta \rho}{D t}=c_{0}^{2}(\delta \boldsymbol{v} \cdot \nabla) \rho_{0}-(\delta \boldsymbol{v} \cdot \nabla) p_{0}+\delta c^{2} \frac{D_{0} \rho_{0}}{D t}
\end{gathered}
$$

where $\delta \rho a$ represents a volume velocity generated by a source, and $\delta \boldsymbol{f} / \rho_{0}$ is a force 
density [8]. This corresponds to a singular source in the fluid, which can be useful for modelling purposes. Here $\delta c^{2}$ be the perturbation of the square of sound velocity caused by the sound wave. In order to obtain $\delta c^{2}$, we expand Eq. (18) as

$$
c^{2}=c_{0}^{2}\left(\rho_{0}, S_{0}\right)+\left[\frac{\partial c^{2}(\rho, S)}{\partial \rho}\right] \delta \rho+\left[\frac{\partial c^{2}(\rho, S)}{\partial S}\right] \delta S+\cdots
$$

Since $c_{0}^{2}$ is known from Eq. (18), this equation becomes

$$
\delta c^{2}=\left[\frac{\partial c^{2}(\rho, S)}{\partial \rho}\right] \delta \rho+\left[\frac{\partial c^{2}(\rho, S)}{\partial S}\right] \delta S .
$$

For an ideal gas, Eq. (30) becomes

$$
c_{0}^{2}+\delta c^{2}=\frac{\gamma\left(p_{0}+\delta p\right)}{\left(\rho_{0}+\delta \rho\right)},
$$

and

$$
\delta c^{2}=\frac{\gamma\left(\rho_{0} \delta p-p_{0} \delta \rho\right)}{\left(\rho_{0}+\delta \rho\right) \rho_{0}}=\frac{\gamma \delta p-c_{0}^{2} \delta \rho}{\rho_{0}},
$$

where the first fraction is an exact result and the last is linearized.

It can be seen that the left hand side of Eqs. (13), (14), (24), and (25) are the material derivatives of the variables $\rho_{0}, v_{0}, \delta \rho, \delta v$ while the right hand side terms only have spatial derivatives. So, if the spatial derivative at each moment in time can be computed with the meshfree method, we can solve these variable values corresponding to fluid particles by temporal integration of the material derivative. Following this, the background motion of the fluid particle can be obtained from Eqs. (13), (14), and (17)-(19), and the acoustic variables of each particle can be obtained from Eqs. (27)-(31). In this way, we have a Lagrangian approach to solve the LLAPE.

\subsection{Equations for different cases}

\subsubsection{Sound propagation in steady uniform flow}

The LAPE are now applied to determine sound propagation in steady uniform flow. A general uniform flow ignoring gravity can be represented as

$$
\rho_{0}=\text { constant }, v_{0}=\text { constant }, \nabla p_{0}=0 \text {. }
$$

Substituting into Eqs. (27)-(29), the LLAPE for steady uniform flow become

$$
\frac{D_{0} \delta \rho}{D t}=-\rho_{0} \nabla \cdot \delta v+\delta \rho a
$$




$$
\begin{gathered}
\frac{D_{0} \delta \boldsymbol{v}}{D t}=-\frac{1}{\rho_{0}} \nabla \delta p+\frac{\delta \boldsymbol{f}}{\rho_{0}}, \\
\frac{D_{0} \delta p}{D t}=c_{0}^{2} \frac{D_{0} \delta \rho}{D t} .
\end{gathered}
$$

In quiescent media, Eqs. (35)-(37) leads back to acoustic wave equations.

\subsubsection{Sound propagation in incompressible steady flow}

Let sound waves travel through incompressible steady flow with constant density. Solutions of Eqs. (13), (14), and (17) ignoring gravity are obtained from

$$
\rho_{0}=\text { constant }, \nabla \cdot \boldsymbol{v}_{0}=0, \boldsymbol{v}_{0} \cdot \nabla \boldsymbol{v}_{0}=-\frac{1}{\rho_{0}} \nabla p_{0} .
$$

The corresponding linearized Lagrangian acoustic wave equations for incompressible steady flow are

$$
\begin{gathered}
\frac{D_{0} \delta \rho}{D t}=-\rho_{0} \nabla \cdot \delta \boldsymbol{v}+\delta \rho a \\
\frac{D_{0} \delta \boldsymbol{v}}{D t}=-\frac{1}{\rho_{0}} \nabla \delta p+\frac{\delta \rho}{\left(\rho_{0}\right)^{2}} \nabla p_{0}+\frac{\delta \boldsymbol{f}}{\rho_{0}} \\
\frac{D_{0} \delta p}{D t}+\delta \boldsymbol{v} \nabla p_{0}=c_{0}^{2} \frac{D_{0} \delta \rho}{D t}
\end{gathered}
$$

\section{SPH and CSP Equations}

Since the SPH method is a famous and widely used meshfree method in computational fluid dynamics, the LAPE and LLAPE are solved using SPH and a correction to it, CSP, in this section.

\subsection{Basic concepts of SPH}

SPH is a meshfree Lagrangian particle method. Functions in the SPH method are represented by a particle approximation. Some basic concepts are presented in this section.

A function $f(\boldsymbol{r})$ can be represented as

$$
<f(\boldsymbol{r})>=\int_{\Omega} f\left(\boldsymbol{r}^{\prime}\right) W\left(\boldsymbol{r}-\boldsymbol{r}^{\prime}, h\right) \mathrm{d} \boldsymbol{r}^{\prime},
$$

where $f$ is a function of the vector $r, \Omega$ is the volume of the integral, $W$ is the smoothing kernel, and $h$ is the smoothing length which represents the size of the support domain. The kernel approximation operator is denoted by the angle bracket $<>$.

The particle approximation for the function $f(\boldsymbol{r})$ at particle $i$ can be written as 


$$
<f\left(\boldsymbol{r}_{i}\right)>=\sum_{j=1}^{N} \frac{m_{j}}{\rho_{j}} f\left(\boldsymbol{r}_{j}\right) W_{i j},
$$

where $\boldsymbol{r}_{i}$ and $\boldsymbol{r}_{j}$ are the positions of particles $i$ and $j, N$ is the number of particles in the computational domain, $m_{j}$ is the mass of particle $j, W_{i j}=W\left(\boldsymbol{r}_{i j}, h\right)$ and $\boldsymbol{r}_{i j}$ is the distance between particle $i$ and particle $j$ as shown in Fig. 2.

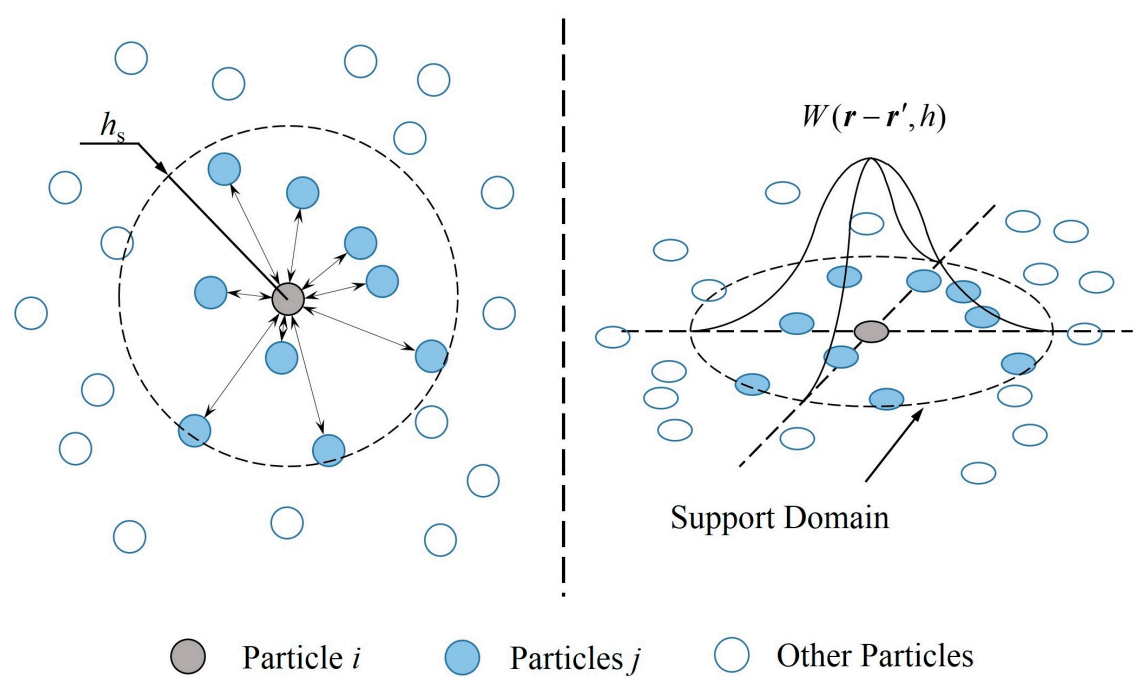

Fig. 2 Fluid particles, support domain and the kernel function.

The spatial derivative $\nabla f\left(\boldsymbol{r}_{i}\right)$ is similarly obtained as

$$
<\nabla f\left(\boldsymbol{r}_{i}\right)>=\sum_{j=1}^{N} \frac{m_{j}}{\rho_{j}} f\left(\boldsymbol{r}_{j}\right) \nabla_{i} W_{i j}
$$

where $\nabla_{i} W_{i j}=\frac{\boldsymbol{r}_{i}-\boldsymbol{r}_{j}}{r_{i j}} \frac{\partial W_{i j}}{\partial r_{i j}}$ and $r_{i j}=\left|\boldsymbol{r}_{i}-\boldsymbol{r}_{j}\right|$. This can also be derived for the divergence of a vector field.

The cubic spline function given by Monaghan and Lattanzio [53], which is widely used in the computational fluid dynamics, is chosen as the smoothing kernel in the present paper.

\subsection{SPH equations for background flow}

Particle approximation for fluid dynamic equations (Eqs. (5), (6), and (9)) in the absence of forces are given in [37]. The particle approximation used for the hydrodynamic equations including gravity (Eqs. (13), (14), and (17)) can be obtained in a similar way. The results are

$$
\frac{D_{0} \rho_{i}}{D t}=\rho_{i} \sum_{j=1}^{N} \frac{m_{j}}{\rho_{j}} \boldsymbol{v}_{i j} \cdot \nabla_{i} W_{i j}
$$




$$
\begin{gathered}
\frac{D_{0} \boldsymbol{v}_{i}}{D t}=-\sum_{j=1}^{N} m_{j} \frac{p_{i}+p_{j}}{\rho_{i} \rho_{j}} \nabla_{i} W_{i j}+\boldsymbol{g}, \\
\frac{D_{0} p_{i}}{D t}=c_{0}^{2} \frac{D_{0} \rho_{i}}{D t},
\end{gathered}
$$

where $p_{i}, \rho_{i}$, and $\boldsymbol{v}_{i}$ are the pressure, density, and velocity of the fluid particle $i$ in the absence of sound, corresponding to $p_{0}, \rho_{0}$, and $\boldsymbol{v}_{0}$ in section $2, \boldsymbol{v}_{i j}=\boldsymbol{v}_{i}-\boldsymbol{v}_{j}$, and $\nabla_{i}$ denotes the gradient of the kernel taken with respect to the coordinate of particle $i$.

Other forms of (45) and (46) are

$$
\begin{gathered}
\rho_{i}=\sum_{j=1}^{N} m_{j} W_{i j}, \\
\frac{D_{0} \boldsymbol{v}_{i}}{D t}=-\sum_{j=1}^{N} m_{j}\left[\frac{p_{i}}{\left(\rho_{i}\right)^{2}}+\frac{p_{j}}{\left(\rho_{j}\right)^{2}}\right] \nabla_{i} W_{i j}+\boldsymbol{g} .
\end{gathered}
$$

\subsection{LLAPE in SPH form}

Applying the SPH particle approximation equation (Eq. (44)) to the LLAPE (Eqs. (27)(29)) yields

$$
\begin{aligned}
\frac{D_{0} \delta \rho_{i}}{D t} & =-\rho_{i} \sum_{j=1}^{N} \frac{m_{j}}{\rho_{j}} \delta \boldsymbol{v}_{j} \cdot \nabla_{i} W_{i j}-\delta \rho_{i} \sum_{j=1}^{N} \frac{m_{j}}{\rho_{j}} \boldsymbol{v}_{j} \cdot \nabla_{i} W_{i j} \\
& -\delta \boldsymbol{v}_{i} \sum_{j=1}^{N} \frac{m_{j}}{\rho_{j}} \rho_{j} \nabla_{i} W_{i j}+\delta \rho_{i} a_{i} \\
\frac{D_{0} \delta \boldsymbol{v}_{i}}{D t}= & -\frac{1}{\rho_{i}} \sum_{j=1}^{N} \frac{m_{j}}{\rho_{j}} \delta p_{j} \nabla_{i} W_{i j}-\delta \boldsymbol{v}_{i} \cdot \sum_{j=1}^{N} \frac{m_{j}}{\rho_{j}} \boldsymbol{v}_{j} \cdot \nabla_{i} W_{i j} \\
& +\frac{\delta \rho_{i}}{\left(\rho_{i}\right)^{2}} \sum_{j=1}^{N} \frac{m_{j}}{\rho_{j}} p_{j} \nabla_{i} W_{i j}+\frac{\delta \boldsymbol{f}_{i}}{\rho_{i}} \\
\frac{D_{0} \delta p_{i}}{D t}-c_{0}^{2} \frac{D_{0} \delta \rho_{i}}{D t}= & c_{0}^{2} \delta \boldsymbol{v}_{i} \cdot \sum_{j=1}^{N} \frac{m_{j}}{\rho_{j}} \rho_{j} \nabla_{i} W_{i j}-\delta \boldsymbol{v}_{i} \cdot \sum_{j=1}^{N} \frac{m_{j}}{\rho_{j}} p_{j} \nabla_{i} W_{i j}+\delta c^{2} \frac{D_{0} \rho_{i}}{D t}
\end{aligned}
$$

where $\delta p_{i}, \delta \rho_{i}$, and $\delta v_{i}$ are the perturbations of pressure, density, and velocity of the fluid particle $i$ due to acoustic waves.

Considering the particle approximation of the gradient of the unity and the transformation method presented in [37], the particle approximation equations of the continuity and momentum equations of acoustic waves (Eqs. (50) and (51)) can be rewritten 
as

$$
\begin{aligned}
\frac{D_{0} \delta \rho_{i}}{D t} & =\rho_{i} \sum_{j=1}^{N} \frac{m_{j}}{\rho_{j}} \delta \boldsymbol{v}_{i j} \cdot \nabla_{i} W_{i j}+\delta \rho_{i} \sum_{j=1}^{N} \frac{m_{j}}{\rho_{j}} \boldsymbol{v}_{i j} \cdot \nabla_{i} W_{i j} \\
& -\delta \boldsymbol{v}_{i} \cdot \sum_{j=1}^{N} \frac{m_{j}}{\rho_{j}} \rho_{j} \nabla_{i} W_{i j}+\delta \rho_{i} a_{i} \\
\frac{D_{0} \delta \boldsymbol{v}_{i}}{D t}= & -\sum_{j=1}^{N} m_{j} \frac{\delta p_{i}+\delta p_{j}}{\rho_{i} \rho_{j}} \nabla_{i} W_{i j}+\delta \boldsymbol{v}_{i} \sum_{j=1}^{N} \frac{m_{j}}{\rho_{j}} \boldsymbol{v}_{i j} \cdot \nabla_{i} W_{i j} \\
+ & \frac{\delta \rho_{i}}{\rho_{i}} \sum_{j=1}^{N} m_{j} \frac{p_{i}+p_{j}}{\rho_{i} \rho_{j}} \nabla_{i} W_{i j}+\frac{\delta \boldsymbol{f}_{i}}{\rho_{i}}
\end{aligned}
$$

For sound propagation in steady uniform flow, the SPH form of the LLAPE is

$$
\begin{gathered}
\frac{D_{0} \delta \rho_{i}}{D t}=\rho_{i} \sum_{j=1}^{N} \frac{m_{j}}{\rho_{j}} \delta \boldsymbol{v}_{i j} \cdot \nabla_{i} W_{i j}+\delta \rho_{i} a_{i} \\
\frac{D_{0} \delta \boldsymbol{v}_{i}}{D t}=-\sum_{j=1}^{N} m_{j} \frac{\delta p_{i}+\delta p_{j}}{\rho_{i} \rho_{j}} \nabla_{i} W_{i j}+\frac{\delta \boldsymbol{f}_{i}}{\rho_{i}} \\
\frac{D_{0} \delta p_{i}}{D t}=c_{0}^{2} \frac{D_{0} \delta \rho_{i}}{D t}
\end{gathered}
$$

\subsection{CSP method}

The CSP method improve the particle approximation in the standard SPH method using Taylor series.

The Taylor series expansion of a function $f(\boldsymbol{r})$ at a position $\boldsymbol{\xi}$ can be represented as

$$
f(\boldsymbol{r})=f(\boldsymbol{\xi})+\left(\boldsymbol{r}^{\alpha}-\boldsymbol{\xi}^{\alpha}\right) f_{\alpha}(\boldsymbol{\xi})+\frac{1}{2 !}\left(\boldsymbol{r}^{\alpha}-\boldsymbol{\xi}^{\alpha}\right)\left(\boldsymbol{r}^{\beta}-\boldsymbol{\xi}^{\beta}\right) f_{\alpha \beta}(\boldsymbol{\xi})+\ldots
$$

where $\alpha, \beta=1,2,3$ are the dimension and

$$
\begin{gathered}
f_{\alpha}(\boldsymbol{\xi})=\frac{\partial f(\boldsymbol{\xi})}{\partial \boldsymbol{r}^{\alpha}}, \\
f_{\alpha \beta}(\boldsymbol{\xi})=\frac{\partial^{2} f(\boldsymbol{\xi})}{\partial \boldsymbol{r}^{\alpha} \partial \boldsymbol{r}^{\beta}} .
\end{gathered}
$$

Multiplying both sides of Eq. (58) by the smoothing function $W(\boldsymbol{r}-\boldsymbol{\xi})$ yields

$$
\begin{aligned}
\int_{\Omega} f(\boldsymbol{r}) W(\boldsymbol{r}-\boldsymbol{\xi}, h) d \boldsymbol{r} & =\int_{\Omega} f(\boldsymbol{\xi}) W(\boldsymbol{r}-\boldsymbol{\xi}, h) d \boldsymbol{r}+\int_{\Omega}\left(\boldsymbol{r}^{\alpha}-\boldsymbol{\xi}^{\alpha}\right) f_{\alpha}(\boldsymbol{\xi}) W(\boldsymbol{r}-\boldsymbol{\xi}, h) d \boldsymbol{r} \\
& +\int_{\Omega} \frac{1}{2 !}\left(\boldsymbol{r}^{\alpha}-\boldsymbol{\xi}^{\alpha}\right)\left(\boldsymbol{r}^{\beta}-\boldsymbol{\xi}^{\beta}\right) f_{\alpha \beta}(\boldsymbol{\xi}) W(\boldsymbol{r}-\boldsymbol{\xi}, h) d \boldsymbol{r}+\ldots
\end{aligned}
$$

Ignoring all derivative terms, $f(\xi)$ can be represented as 


$$
<f(\boldsymbol{\xi})>=\frac{\int_{\Omega} f(\boldsymbol{r}) W(\boldsymbol{r}-\boldsymbol{\xi}, h) d \boldsymbol{r}}{\int_{\Omega} W(\boldsymbol{r}-\boldsymbol{\xi}, h) d \boldsymbol{r}} .
$$

Multiplying both sides of Eq. (58) with $\nabla W(\boldsymbol{r}-\boldsymbol{\xi})$ yields

$$
\begin{aligned}
\int_{\Omega} f(\boldsymbol{r}) \nabla W(\boldsymbol{r}-\boldsymbol{\xi}, h) d \boldsymbol{r} & =\int_{\Omega} f(\boldsymbol{\xi}) \nabla W(\boldsymbol{r}-\boldsymbol{\xi}, h) d \boldsymbol{r} \\
& +\int_{\Omega}\left(\boldsymbol{r}^{\alpha}-\boldsymbol{\xi}^{\alpha}\right) f_{\alpha}(\boldsymbol{\xi}) \nabla W(\boldsymbol{r}-\boldsymbol{\xi}, h) d \boldsymbol{r} \\
& +\int_{\Omega} \frac{1}{2 !}\left(\boldsymbol{r}^{\alpha}-\boldsymbol{\xi}^{\alpha}\right)\left(\boldsymbol{r}^{\beta}-\boldsymbol{\xi}^{\beta}\right) f_{\alpha \beta}(\boldsymbol{\xi}) \nabla W(\boldsymbol{r}-\boldsymbol{\xi}, h) d \boldsymbol{r} \\
& +\ldots
\end{aligned}
$$

where

$$
\nabla W(\boldsymbol{r}-\boldsymbol{\xi}, h)=\frac{\partial W(\boldsymbol{r}-\boldsymbol{\xi}, h)}{\partial \boldsymbol{r}} .
$$

Neglecting second and higher derivative terms, $f_{\alpha}(\xi)$ can be represented as

$$
<f_{\alpha}(\boldsymbol{\xi})>=\frac{\int_{\Omega}[f(\boldsymbol{r})-f(\boldsymbol{\xi})] \nabla W(\boldsymbol{r}-\boldsymbol{\xi}, h) d \boldsymbol{r}}{\int_{\Omega}\left(\boldsymbol{r}^{\alpha}-\boldsymbol{\xi}^{\alpha}\right) \nabla W(\boldsymbol{r}-\boldsymbol{\xi}, h) d \boldsymbol{r}} .
$$

Following a similar derivation to that in the standard SPH method, the particle approximations for Eqs. (62) and (65) at particle $i$ become

$$
\begin{gathered}
f\left(\boldsymbol{r}_{i}\right)=\frac{\sum_{j=1}^{N} \frac{m_{j}}{\rho_{j}} f\left(\boldsymbol{r}_{j}\right) W_{i j}}{\sum_{j=1}^{N} \frac{m_{j}}{\rho_{j}} W_{i j}}, \\
f_{\alpha}\left(\boldsymbol{r}_{i}\right)=\frac{\sum_{j=1}^{N} \frac{m_{j}}{\rho_{j}}\left[f\left(\boldsymbol{r}_{j}\right)-f\left(\boldsymbol{r}_{i}\right)\right] \nabla_{i} W_{i j}}{\sum_{j=1}^{N} \frac{m_{j}}{\rho_{j}}\left(\boldsymbol{r}_{j}^{\alpha}-\boldsymbol{r}_{i}^{\alpha}\right) \nabla_{i} W_{i j}} .
\end{gathered}
$$

\subsection{Particle motion and time integration}

In our Lagrangian approach, calculation of the particle motion and time integration is performed with a second-order leapfrog integration [54]. In this scheme, the equations for updating the position of particles and the velocity are

$$
v_{i}\left(t+\frac{1}{2} \Delta t\right)=v_{i}\left(t-\frac{1}{2} \Delta t\right)+\Delta t \frac{D_{0} v_{i}(t)}{D t},
$$




$$
\boldsymbol{r}_{i}(t+\Delta t)=\boldsymbol{r}_{i}(t)+\Delta t \boldsymbol{v}_{i}\left(t+\frac{1}{2} \Delta t\right)
$$

where $v_{i}\left(t+\frac{1}{2} \Delta t\right)$ is the velocity of fluid particle $i$ at time $t+\frac{1}{2} \Delta t, t$ is the time of previous timestep, and $\Delta t$ is the time step.

Eq. (68) starts with the initial velocity offset given by

$$
v_{i}\left(\frac{1}{2} \Delta t\right)=v_{i}(0)+\frac{1}{2} \Delta t \frac{D_{0} v_{i}(0)}{D t}
$$

\section{GFD Equations}

The consistency problem always limit the accuracy of SPH method [21, 55], and the gradient of kernel function have some special requirements on selecting a kernel function [37]. So, in this section, we show another meshfree method, namely the GFD method, which only use the kernel itself. This method reduces to standard finite difference methods (FDM) on an orthogonal grid, and can be easily extended to higher orders [56].

\subsection{Spatial derivative approximation by GFD}

Consider a particle $i$ surrounded by particles $j=1,2, \ldots, N$, with all $N+1$ of them are in a compact support domain. The value of an infinitely differentiable function $F$ at the position of particles $i$ and $j$ are defined as $F_{i}$ and $F_{j}$. Let us expand in the Taylor series of $F$ at the particle $i$ :

$$
\begin{gathered}
F_{j}=F_{i}+h_{j} \frac{\partial F_{i}}{\partial x}+\frac{h_{j}^{2}}{2} \frac{\partial^{2} F_{i}}{\partial x^{2}}+\cdots \\
F_{j}=F_{i}+h_{j} \frac{\partial F_{i}}{\partial x}+k_{j} \frac{\partial F_{i}}{\partial y}+\frac{h_{j}^{2}}{2} \frac{\partial^{2} F_{i}}{\partial x^{2}}+\frac{k_{j}^{2}}{2} \frac{\partial^{2} F_{i}}{\partial y^{2}}+h_{j} k_{j} \frac{\partial^{2} F_{i}}{\partial x \partial y}+\cdots,
\end{gathered}
$$

where Eqs. (71) and (72) are for 1D and 2D, respectively, with $h_{j}=x_{j}-x_{i}$, and $k_{j}=y_{j}-y_{i}$.

Ignoring higher order terms, the approximation of $F$ is denoted by $f$ :

$$
\begin{gathered}
f_{j}=f_{i}+h_{j} \frac{\partial f_{i}}{\partial x}+\frac{h_{j}^{2}}{2} \frac{\partial^{2} f_{i}}{\partial x^{2}} \\
f_{j}=f_{i}+h_{j} \frac{\partial f_{i}}{\partial x}+k_{j} \frac{\partial f_{i}}{\partial y}+\frac{h_{j}^{2}}{2} \frac{\partial^{2} f_{i}}{\partial x^{2}}+\frac{k_{j}^{2}}{2} \frac{\partial^{2} f_{i}}{\partial y^{2}}+h_{j} k_{j} \frac{\partial^{2} f_{i}}{\partial x \partial y} .
\end{gathered}
$$

After rearranging these equations, the sum of these expressions for all particles $j$ with multiplying weight functions are obtained: 


$$
\begin{gathered}
\sum_{j=1}^{N}\left(f_{i}-f_{j}+h_{j} \frac{\partial f_{i}}{\partial x}+\frac{h_{j}^{2}}{2} \frac{\partial^{2} f_{i}}{\partial x^{2}}\right) W\left(h_{j}, h\right)=0, \\
\sum_{j=1}^{N}\left(\begin{array}{l}
f_{i}-f_{j}+h_{j} \frac{\partial f_{i}}{\partial x}+k_{j} \frac{\partial f_{i}}{\partial y} \\
+\frac{h_{j}^{2}}{2} \frac{\partial^{2} f_{i}}{\partial x^{2}}+\frac{k_{j}^{2}}{2} \frac{\partial^{2} f_{i}}{\partial y^{2}}+h_{j} k_{j} \frac{\partial^{2} f_{i}}{\partial x \partial y}
\end{array}\right) W\left(h_{j}, k_{j}, h\right)=0,
\end{gathered}
$$

where $W\left(h_{j}, h\right)$ and $W\left(h_{j}, k_{j}, h\right)$ are weight functions in 1D and 2D respectively, and $h$ represents the size of the support domain, which is always called smoothing length in the SPH method (see section 3.1). For a circular shape domain, $h$ stands for the radius of the support domain.

In order to compare with the SPH method, the closest nodes to the particle $i$ are selected as particles $j$, and these particles should be in the support domain at the same time. In addition, cubic splines are selected in both SPH, CSP, and GFD methods as the weighting function. A comparison of different criterions of particle selection and different weighting functions in the GFD method is given in [47]. In the following equations, we use $W$ for the weighting function.

Defining functions $B_{2}$ in $1 \mathrm{D}$ and $B_{5}$ in 2D as

$$
\begin{gathered}
B_{2}(f)=\sum_{j=1}^{N}\left[\left(f_{i}-f_{j}+h_{j} \frac{\partial f_{i}}{\partial x}+\frac{h_{j}^{2}}{2} \frac{\partial^{2} f_{i}}{\partial x^{2}}\right) W\right]^{2}, \\
B_{5}(f)=\sum_{j=1}^{N}\left[\left(\begin{array}{l}
f_{i}-f_{j}+h_{j} \frac{\partial f_{i}}{\partial x}+k_{j} \frac{\partial f_{i}}{\partial y} \\
\left.+\frac{h_{j}^{2}}{2} \frac{\partial^{2} f_{i}}{\partial x^{2}}+\frac{k_{j}^{2}}{2} \frac{\partial^{2} f_{i}}{\partial y^{2}}+h_{j} k_{j} \frac{\partial^{2} f_{i}}{\partial x \partial y}\right)^{2}
\end{array}\right]^{2} .\right.
\end{gathered}
$$

According to Eqs. (75) and (76), the norms $B_{2}$ and $B_{5}$ equal to 0 , so we obtain:

$$
\begin{aligned}
& \frac{\partial B_{2}(f)}{\partial\left(\frac{\partial f_{i}}{\partial x}\right)}=2 \sum_{j=1}^{N} \Phi_{2} h_{j} W^{2}=0, \\
& \frac{\partial B_{2}(f)}{\partial\left(\frac{\partial^{2} f_{i}}{\partial x^{2}}\right)}=\sum_{j=1}^{N} \Phi_{2} h_{j}^{2} W^{2}=0,
\end{aligned}
$$

and

$$
\begin{aligned}
& \frac{\partial B_{5}(f)}{\partial\left(\frac{\partial f_{i}}{\partial x}\right)}=2 \sum_{j=1}^{N} \Phi_{5} h_{j} W^{2}=0, \\
& \frac{\partial B_{5}(f)}{\partial\left(\frac{\partial f_{i}}{\partial y}\right)}=2 \sum_{j=1}^{N} \Phi_{5} k_{j} W^{2}=0,
\end{aligned}
$$




$$
\begin{gathered}
\frac{\partial B_{5}(f)}{\partial\left(\frac{\partial^{2} f_{i}}{\partial x^{2}}\right)}=\sum_{j=1}^{N} \Phi_{5} h_{j}^{2} W^{2}=0, \\
\frac{\partial B_{5}(f)}{\partial\left(\frac{\partial^{2} f_{i}}{\partial y^{2}}\right)}=\sum_{j=1}^{N} \Phi_{5} k_{j}^{2} W^{2}=0, \\
\frac{\partial B_{5}(f)}{\partial\left(\frac{\partial^{2} f_{i}}{\partial x \partial y}\right)}=2 \sum_{j=1}^{N} \Phi_{5} h_{j} k_{j} W^{2}=0,
\end{gathered}
$$

where

$$
\begin{gathered}
\Phi_{2}=f_{i}-f_{j}+h_{j} \frac{\partial f_{i}}{\partial x}+\frac{h_{j}^{2}}{2} \frac{\partial^{2} f_{i}}{\partial x^{2}} \\
\Phi_{5}=f_{i}-f_{j}+h_{j} \frac{\partial f_{i}}{\partial x}+k_{j} \frac{\partial f_{i}}{\partial y}+\frac{h_{j}^{2}}{2} \frac{\partial^{2} f_{i}}{\partial x^{2}}+\frac{k_{j}^{2}}{2} \frac{\partial^{2} f_{i}}{\partial y^{2}}+h_{j} k_{j} \frac{\partial^{2} f_{i}}{\partial x \partial y} .
\end{gathered}
$$

Eqs. (79)-(85) give us the following equations:

$$
\begin{aligned}
& \mathbf{A}_{2} \mathbf{D}_{\mathrm{u} 2}=\mathbf{b}_{2}, \\
& \mathbf{A}_{5} \mathbf{D}_{\mathrm{u} 5}=\mathbf{b}_{5},
\end{aligned}
$$

where

$$
\begin{aligned}
& \mathbf{A}_{2}=\left[\begin{array}{cc}
\sum_{j=1}^{N} h_{j}^{2} W^{2} & \sum_{j=1}^{N} \frac{1}{2} h_{j}^{3} W^{2} \\
\sum_{j=1}^{N} \frac{1}{2} h_{j}^{3} W^{2} & \sum_{j=1}^{N} \frac{1}{4} h_{j}^{4} W^{2}
\end{array}\right],
\end{aligned}
$$

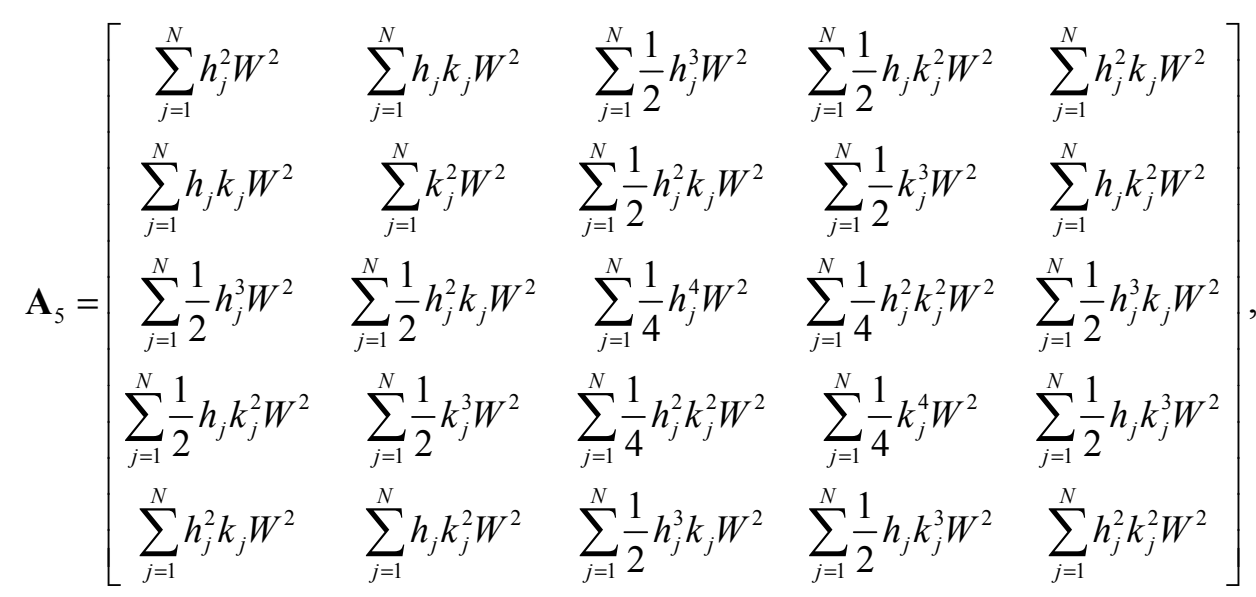

$$
\begin{aligned}
& \mathbf{D}_{\mathrm{u} 2}=\left\{\frac{\partial f_{i}}{\partial x}, \frac{\partial^{2} f_{i}}{\partial x^{2}}\right\}^{\mathrm{T}},
\end{aligned}
$$




$$
\begin{aligned}
& \mathbf{D}_{\mathrm{u} 5}=\left\{\frac{\partial f_{i}}{\partial x}, \frac{\partial f_{i}}{\partial y}, \frac{\partial^{2} f_{i}}{\partial x^{2}}, \frac{\partial^{2} f_{i}}{\partial y^{2}}, \frac{\partial^{2} f_{i}}{\partial x \partial y}\right\}^{\mathrm{T}}, \\
& \mathbf{b}_{2}=\left\{\begin{array}{c}
\sum_{j=1}^{N}\left(f_{j}-f_{i}\right) h_{j} W^{2} \\
\sum_{j=1}^{N}\left(f_{j}-f_{i}\right) \frac{h_{j}^{2}}{2} W^{2}
\end{array}\right\}, \\
& \mathbf{b}_{5}=\left\{\begin{array}{l}
\sum_{j=1}^{N}\left(f_{j}-f_{i}\right) h_{j} W^{2} \\
\sum_{j=1}^{N}\left(f_{j}-f_{i}\right) k_{j} W^{2} \\
\sum_{j=1}^{N}\left(f_{j}-f_{i}\right) \frac{h_{j}^{2}}{2} W^{2} \\
\sum_{j=1}^{N}\left(f_{j}-f_{i}\right) \frac{k_{j}^{2}}{2} W^{2} \\
\sum_{j=1}^{N}\left(f_{j}-f_{i}\right) h_{j} k_{j} W^{2}
\end{array}\right\} .
\end{aligned}
$$

Since the matrices $\mathbf{A}_{2}$ and $\mathbf{A}_{5}$ are symmetrical, Eqs. (88) and (89) can be solved using Cholesky factorization. In this way, the spatial derivative can be obtained and the material derivative in LAPE can be integrated using the time integration scheme.

\subsection{GFD equations for background flow}

The coefficients of $\mathbf{D}_{2}$ and $\mathbf{b}_{2}$ are denoted by $D_{2 m}\left(f_{i}\right)$ and $b_{2 m}\left(f_{i}\right)$ with $m=1,2$, and the coefficients of $\mathbf{D}_{5}$ and $\mathbf{b}_{5}$ are denoted by $D_{5 m}\left(f_{i}\right)$ and $b_{5 m}\left(f_{i}\right)$ with $m=1,2, \ldots 5$. For example, $D_{22}\left(f_{i}\right)=\frac{\partial^{2} f_{i}}{\partial x^{2}}, \quad b_{22}\left(f_{i}\right)=\sum_{j=1}^{N}\left(f_{j}-f_{i}\right) \frac{h_{j}^{2}}{2} W^{2}, D_{52}\left(f_{i}\right)=\frac{\partial f_{i}}{\partial y}$, and $b_{52}\left(f_{i}\right)=\sum_{j=1}^{N}\left(f_{j}-f_{i}\right) k_{j} W^{2}$.

Besides, the matrices $\mathbf{A}_{2}$ and $\mathbf{A}_{5}$ has a decomposition in upper and lower triangular matrices $\mathbf{A}_{2}=\mathbf{L}_{2} \mathbf{L}_{2}^{\mathbf{T}}$ and $\mathbf{A}_{5}=\mathbf{L}_{5} \mathbf{L}_{5}^{\mathbf{T}}$. The coefficients of the matrices $\mathbf{L}_{2}$ and $\mathbf{L}_{5}$ are denoted by $L_{2}(m$, $n$ ) with $m, n=1,2$ and $L_{5}(m, n)$ with $m, n=1,2,3,4,5$. For example, the matrices $\mathbf{A}_{2}$ is written as

$$
\mathbf{A}_{2}=\left(\begin{array}{cc}
L_{2}(1,1) & 0 \\
L_{2}(2,1) & L_{2}(2,2)
\end{array}\right)\left(\begin{array}{cc}
L_{2}(1,1) & L_{2}(2,1) \\
0 & L_{2}(2,2)
\end{array}\right) .
$$

By using the GFD method and Cholesky factorization to solve Eqs. (88) and (89), we obtain the solutions for Eqs. (13) and (14) in one dimension from 


$$
\begin{gathered}
\frac{D_{0} \rho_{i}}{D t}=-\rho_{i} D_{21}\left(u_{i}\right), \\
\frac{D_{0} u_{i}}{D t}=-\frac{1}{\rho_{i}} D_{21}\left(p_{i}\right)+\boldsymbol{g} .
\end{gathered}
$$

In two dimensions, we have

$$
\begin{gathered}
\frac{D_{0} \rho_{i}}{D t}=-\rho_{i} D_{51}\left(u_{i}\right)-\rho_{i} D_{52}\left(v_{i}\right), \\
\frac{D_{0} u_{i}}{D t}=-\frac{1}{\rho_{i}} D_{51}\left(p_{i}\right) \\
\frac{D_{0} v_{i}}{D t}=-\frac{1}{\rho_{i}} D_{52}\left(p_{i}\right)+\boldsymbol{g}
\end{gathered}
$$

where $u_{i}$ and $v_{i}$ are the velocity of particle $i$ along two directions, and

$$
\begin{gathered}
D_{2 i}(f)=\left\{\begin{array}{cc}
\frac{1}{L_{2}(1,1)^{2}}\left[b_{21}(f)-L_{2}(1,1) L_{2}(2,1) D_{22}(f)\right] & i=1 \\
\frac{1}{L_{2}(2,2)^{2}}\left[b_{22}(f)-\frac{L_{2}(2,1) b_{21}(f)}{L_{2}(1,1)}\right] & i=2
\end{array},\right. \\
D_{5 i}(f)=\left\{\begin{array}{cc}
\frac{1}{L_{5}(i, i)}\left[Y_{5 i}(f)-\sum_{j=i+1}^{5} L_{5}(j, i) D_{5 j}(f)\right] & i=1,2,3,4 \\
\frac{Y_{55}(f)}{L_{5}(5,5)} & i=5
\end{array},\right.
\end{gathered}
$$

where

$$
Y_{5 i}(f)=\left\{\begin{array}{cc}
\frac{b_{5 i}(f)}{L_{5}(1,1)} & i=1 \\
\frac{1}{L_{5}(i, i)}\left[b_{5 i}(f)-\sum_{j=1}^{i-1} L_{5}(i, j) Y_{5 j}(f)\right] & i=2,3,4,5
\end{array} .\right.
$$

For an ideal gas, the equation of state is Eq. (20).

\subsection{LLAPE in GFD form}

The GFD form of the LLAPE (Eqs. (27) and (28)) ignoring the force density is given in this section as an example. The GFD formulation in one dimension is

$$
\begin{gathered}
\frac{D_{0} \delta \rho_{i}}{D t}=-\rho_{i} D_{21}\left(\delta u_{i}\right)-\delta \rho_{i} D_{21}\left(u_{i}\right)-\delta u_{i} D_{21}\left(\rho_{i}\right)+\delta \rho_{i} a_{i}, \\
\frac{D_{0} \delta u_{i}}{D t}=-\frac{1}{\rho_{i}} D_{21}\left(\delta p_{i}\right)-\delta u_{i} D_{21}\left(u_{i}\right)+\frac{\delta \rho_{i}}{\rho_{i}^{2}} D_{21}\left(p_{i}\right),
\end{gathered}
$$




$$
\frac{D_{0} \delta p_{i}}{D t}-c_{0}^{2} \frac{D_{0} \delta \rho_{i}}{D t}=c_{0}^{2} \delta u_{i} D_{21}\left(\rho_{i}\right)-\delta u_{i} D_{21}\left(p_{i}\right)-\delta c^{2} \rho_{i} D_{21}\left(u_{i}\right)
$$

In two dimensions, we have

$$
\begin{gathered}
\frac{D_{0} \delta \rho_{i}}{D t}=-\rho_{i}\left[D_{51}\left(\delta u_{i}\right)+D_{52}\left(\delta v_{i}\right)\right]-\delta \rho_{i}\left[D_{51}\left(u_{i}\right)+D_{52}\left(v_{i}\right)\right] \\
-\delta u_{i} D_{51}\left(\rho_{i}\right)-\delta v_{i} D_{52}\left(\rho_{i}\right)+\delta \rho_{i} a_{i} \\
\frac{D_{0} \delta u_{i}}{D t}=-\frac{1}{\rho_{i}} D_{51}\left(\delta p_{i}\right)-\delta u_{i} D_{51}\left(u_{i}\right)-\delta v_{i} D_{52}\left(u_{i}\right)+\frac{\delta \rho_{i}}{\rho_{i}^{2}} D_{51}\left(p_{i}\right) \\
\begin{aligned}
\frac{D_{0} \delta v_{i}}{D t}=-\frac{1}{\rho_{i}} D_{52}\left(\delta p_{i}\right)- & \delta u_{i} D_{51}\left(v_{i}\right)-\delta v_{i} D_{52}\left(v_{i}\right)+\frac{\delta \rho_{i}}{\rho_{i}^{2}} D_{52}\left(p_{i}\right) \\
\frac{D_{0} \delta p_{i}}{D t}-c_{0}^{2} \frac{D_{0} \delta \rho_{i}}{D t}= & c_{0}^{2}\left[\delta u_{i} D_{51}\left(\rho_{i}\right)+\delta v_{i} D_{52}\left(\rho_{i}\right)\right] \\
& -\delta u_{i} D_{51}\left(p_{i}\right)-\delta v_{i} D_{52}\left(p_{i}\right) \\
& -\delta c^{2} \rho_{i}\left[D_{51}\left(u_{i}\right)+D_{52}\left(v_{i}\right)\right]
\end{aligned}
\end{gathered}
$$

where $\delta u_{i}$ and $\delta v_{i}$ are the velocity perturbation of particle $i$ along two directions. For an ideal gas, the equation of state is Eq. (33).

\section{Numerical Tests and Discussion}

In this section, different test cases with applications to modeling sound propagation in moving fluids are outlined.

5.1 1D Sound propagation in steady uniform flow

Sound propagation in steady uniform background flow is examined in this section. The test example is originally provided in the Fourth Computational Aeroacoustics Workshop on Benchmark Problems [57]. Consider sound propagation in a 1D homogeneous, steady uniform moving medium. The initial conditions are

$$
\begin{gathered}
\delta u(x, t=0)=\alpha_{1} c_{0}[2+\cos (k x)] \exp \left[-\alpha_{2}(k x)^{2}\right], \\
\delta p(x, t=0)=\alpha_{1} \rho_{0} c_{0}^{2}[2+\cos (k x)] \exp \left[-\alpha_{2}(k x)^{2}\right], \\
\delta \rho(x, t=0)=\alpha_{1} \rho_{0}[2+\cos (k x)] \exp \left[-\alpha_{2}(k x)^{2}\right]
\end{gathered}
$$

where $\delta u$ is the velocity perturbation along the $x$-axis, $t$ represents time, $x$ is the geometric position, $k$ is the wave number, and $\alpha_{1}=4 / 340^{2}, \alpha_{2}=\ln 2 / 120$. 
The fluid is an ideal gas with sound speed $c_{0} 340 \mathrm{~m} / \mathrm{s}$ and density $1.0 \mathrm{~kg} / \mathrm{m}^{3}$. The exact solution for the LLAPE at time $t$ is

$$
\begin{gathered}
\delta u(x, t)=\alpha_{1} c_{0}[2+\cos (k x-w t)] \exp \left[-\alpha_{2}(k x-w t)^{2}\right] \\
\delta p(x, t)=\alpha_{1} \rho_{0} c_{0}^{2}[2+\cos (k x-w t)] \exp \left[-\alpha_{2}(k x-w t)^{2}\right] \\
\delta \rho(x, t)=\alpha_{1} \rho_{0}[2+\cos (k x-w t)] \exp \left[-\alpha_{2}(k x-w t)^{2}\right] .
\end{gathered}
$$

In the model, sound waves propagate along the $x$-axis over a time interval of $0.5 \mathrm{~s}, w$ is the angular frequency of $340 \mathrm{rad} / \mathrm{s}$, and $k$ is set as $1.0 \mathrm{rad} / \mathrm{m}$. The computational domain spans $-250 \mathrm{~m}$ to $250 \mathrm{~m}$. Since the computational region is far longer than the sound propagation distance, Dirichlet boundary conditions are applied to both ends of the computational region. The sound pressure and perturbations of density and velocity for the last three particles at each end are set as 0 , which are their initial value.

In the computation, the particle spacing $(\Delta x)$ is set to be $0.05 \mathrm{~m}$, the Courant-FriedrichsLewy (CFL) number is 0.15 , and the smoothing length $(h)$ for the simulation is $1.45 \Delta x$. The CFL number is defined as

$$
\Delta t=C_{\mathrm{CFL}} \frac{\Delta x}{c_{0}}
$$

where $\Delta x$ is the time step and $C_{\mathrm{CFL}}$ is the CFL number.

CSP results at time $t$ are compared with exact solutions in Fig. 3, and three test cases with the moving medium at different Mach numbers are shown. Computational results are plotted with points at intervals of 5 grid points, while exact solutions are plotted with solid lines. The Mach number of the background flow changes from -0.2 to 0.2 . Results from the SPH and GFD methods are not given here because they are so similar to the CSP method that the difference cannot be distinguished in the figure. 


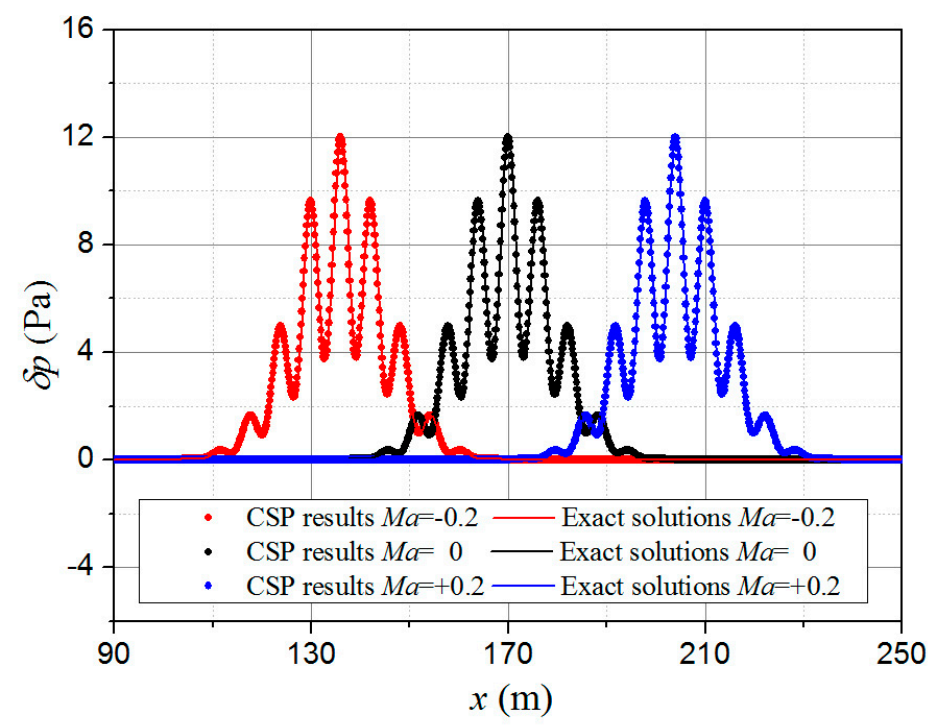

Fig. 3 Sound pressure comparison between CSP and exact solutions for the LLAPE with the moving medium at different Mach numbers.

The figure shows that sound pressure amplitude and tendency obtained by the CSP method agree well with exact solutions, and the CSP method is validated for this problem.

To evaluate the convergence of the three meshfree methods, a non-dimensional sound pressure error $\left(\varepsilon_{\text {pre }}\right)$ is used to discuss the effects of $\Delta x$ and $C_{\mathrm{CFL}} . \varepsilon_{\text {pre }}$ is defined as:

$$
\varepsilon_{\text {pre }}=\frac{1}{3 \alpha_{1} \rho_{0} c_{0}^{2} N} \sqrt{\sum_{j=1}^{N}\left[p_{j}^{*}-p\left(x_{j}\right)\right]^{2}},
$$

where $N$ is the number of particles in the computational domain, $p_{j}^{*}$ is the numerical solutions of sound pressure for particle $j, p\left(x_{j}\right)$ is the theoretical sound pressure at the position $x_{j}$, and $x_{j}$ is the position of particle $j$.

Convergence and CPU time ( $\left.t_{\mathrm{CPU}}\right)$ curves for SPH, CSP, and GFD solutions of LLAPE are shown in Fig. 4 and Fig. 5. The exact solutions for LLAPE come from Eqs. (115)-(117). All computational parameters stay the same as in the model for Fig. 3 except $\Delta x$. All computation in the present paper were performed with processor Intel(R) Core(TM) i74710MQ CPU 2.50 GHz and RAM 8.00 GB. According to our computations, the Mach number has little effects on the error, so only one Mach number is used in the following comparison. 


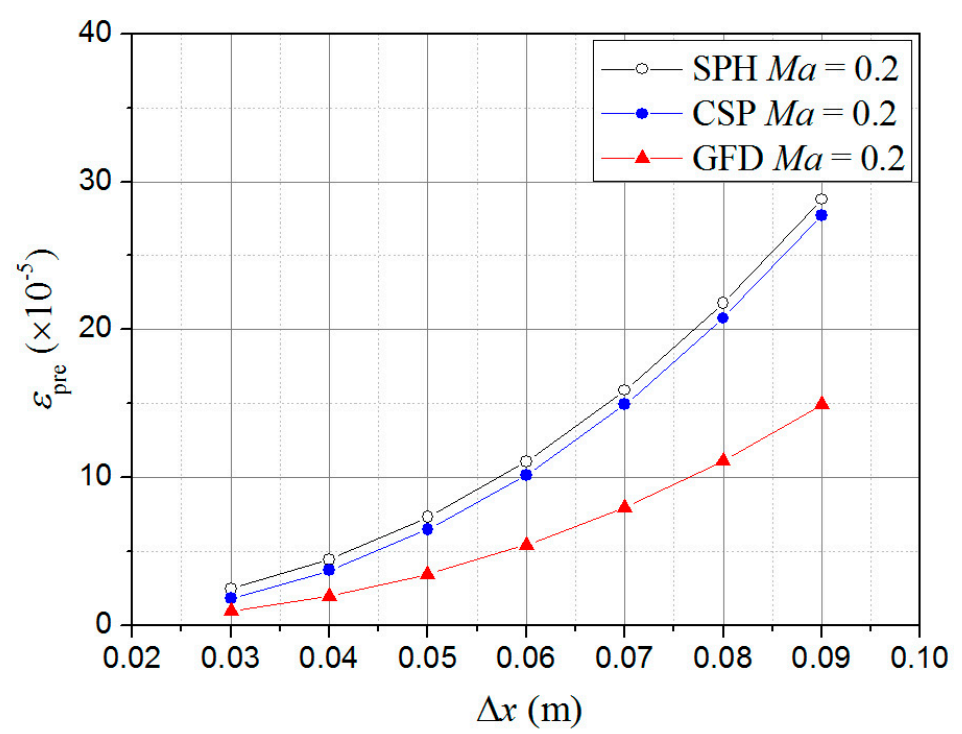

Fig. 4 Convergence curves for SPH, CSP, and GFD solutions of LLAPE at $M a=0.2$.

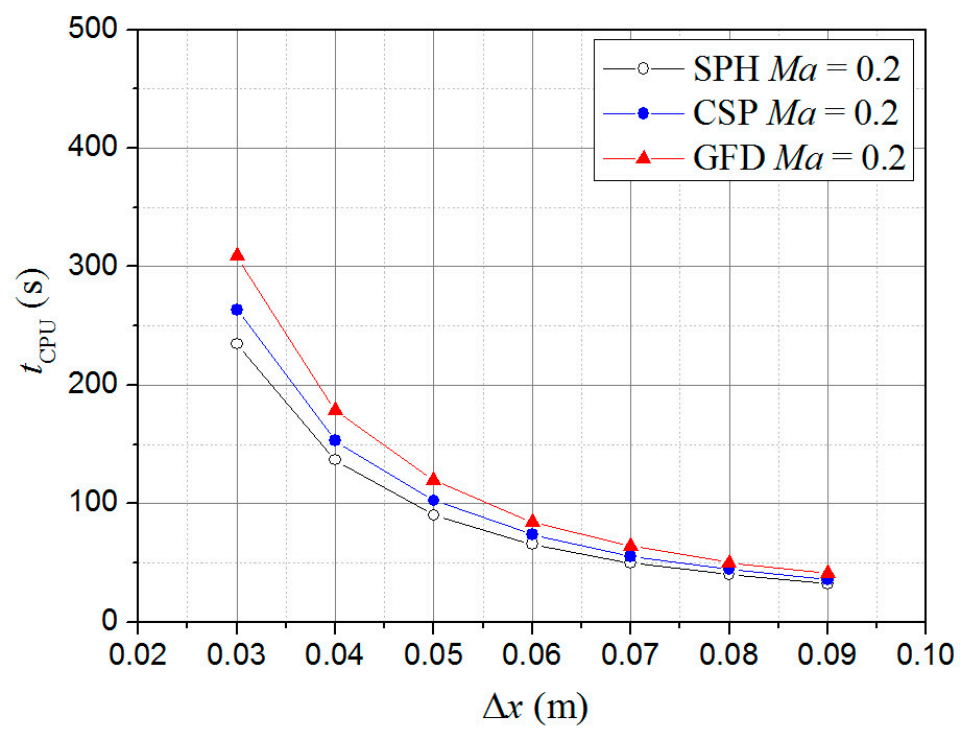

Fig. 5 CPU time curves for SPH, CSP, and GFD solutions of LLAPE at $M a=0.2$ at different $\Delta x$.

As shown in Fig. 4, all three meshfree methods are convergent for this problem. The GFD method has better accuracy for different $\Delta x$ compared with the other two methods, while the SPH and CSP methods are similar. As $\Delta x$ increases from 0.03 to $0.09 \mathrm{~m}, \varepsilon$ pre for the GFD method increases from around $1.0 \times 10^{-5}$ to $1.5 \times 10^{-4}$, and $\varepsilon$ pre for the SPH and CSP methods grow from about $2.0 \times 10^{-5}$ to over $2.5 \times 10^{-4}$. However, the decrease of $\Delta x$ not only brings accuracy improvements, but also increases the time of computation according to Fig. 5 . The GFD method has the highest time cost, while the SPH method has the lowest cost. The graph shows a fivefold increases for all three methods in $t_{\mathrm{CPU}}$ when $\Delta x$ decreases from 0.09 to 0.03 m.

The effects of $C_{\mathrm{CFL}}$ on $\varepsilon_{\text {pre }}$ and $t_{\mathrm{CPU}}$ for the three meshfree methods solving the LLAPE at $M a=0.2$ are shown in Fig. 6 and Fig. 7. All computational parameters are the same as in 
the computation for Fig. 3 with only $C_{\mathrm{CFL}}$ changing.

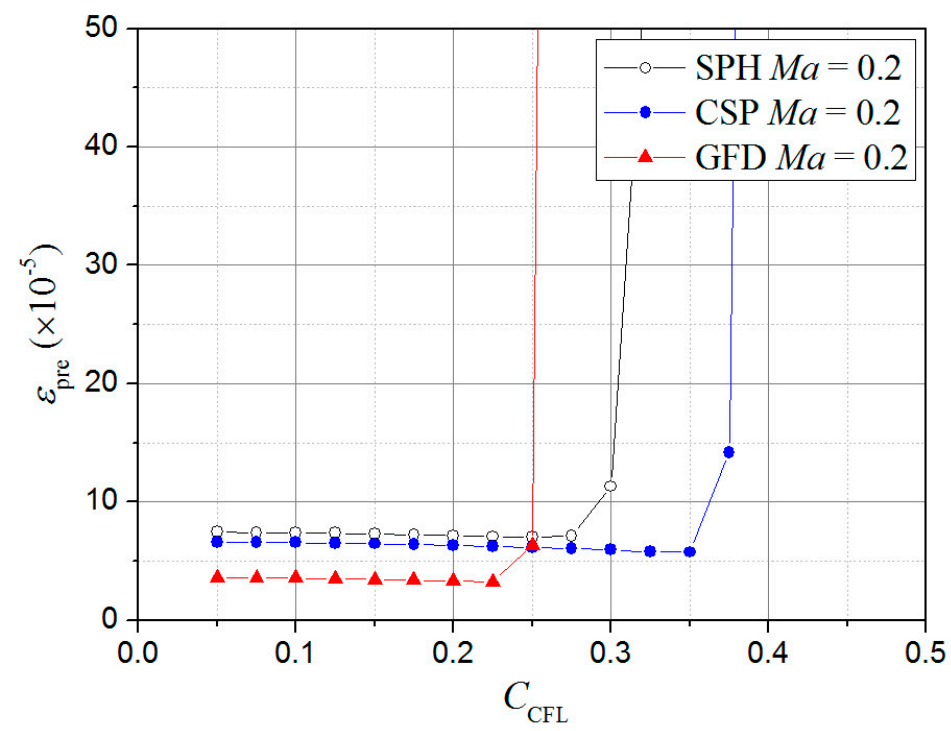

Fig. $6 \varepsilon_{\text {pre }}$ for SPH, CSP, and GFD solutions of LLAPE at $M a=0.2$ with different $C_{\mathrm{CFL}}$.

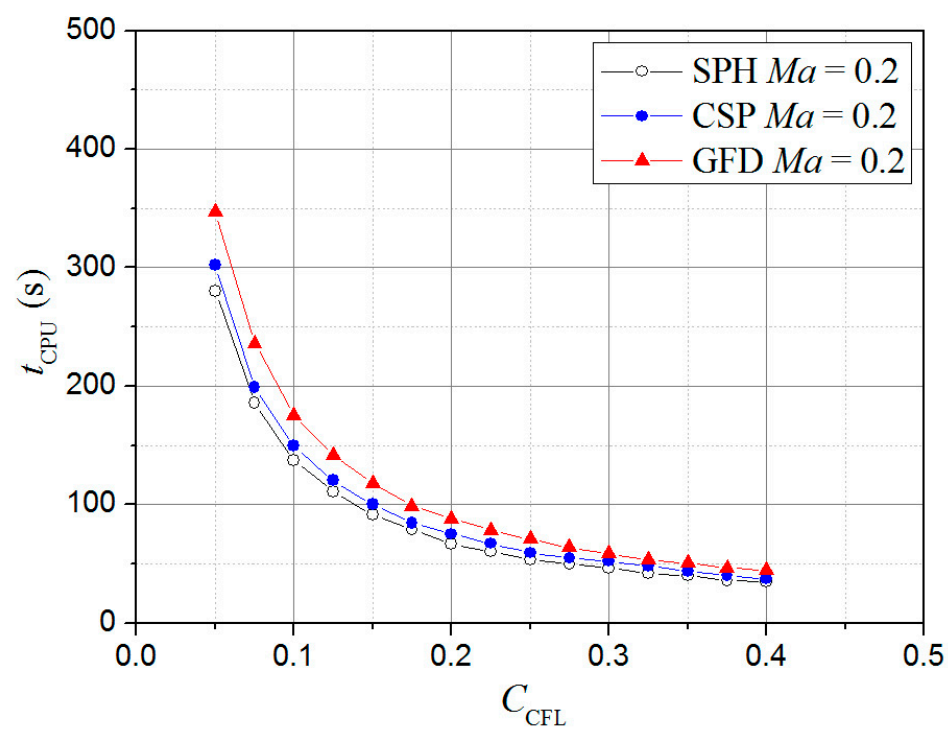

Fig. 7 CPU time curves for SPH, CSP, and GFD solutions of LLAPE at $M a=0.2$ with different $C_{\mathrm{CFL}}$.

Fig. 6 shows that accurate numerical results depend on an appropriate choice of $C_{\text {CFL }}$. When $C_{\text {CFL }}$ is small enough, $\varepsilon$ pre remains small. As $C_{\text {CFL }}$ increases, there is a rapid growth in $\varepsilon$ pre. The value of $C_{\mathrm{CFL}}$ to keep the computation stable is different for the three methods.

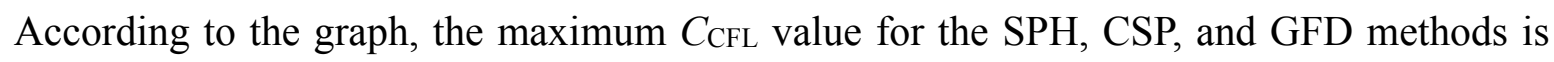

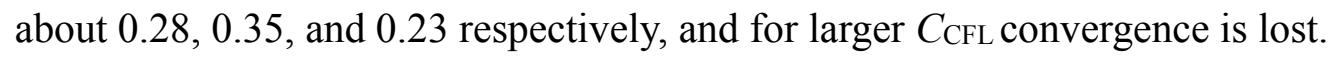

Fig. 7 shows that small $C_{\mathrm{CFL}}$ leasds to noticeable growth in $t_{\mathrm{CPU}}$, and the GFD method requires more $t_{\mathrm{CPU}}$ than other two methods. For the three mehfree methods, as $C_{\mathrm{CFL}}$ changes from 0.05 to $0.4, t_{\mathrm{CPU}}$ decreases from around $300 \mathrm{~s}$ to $50 \mathrm{~s}$. When $C_{\mathrm{CFL}}$ is small, this tendency is more obvious then at large $C_{\mathrm{CFL}}$.

The effects of $h / \Delta x$ on numerical solutions of LLAPE at $M a=0.2$ are shown in Fig. 8. 
In the test, $h / \Delta x$ changes from 1.2 to 1.7 corresponding to 4 particles in the support domain, which is suggested for the SPH method [37].

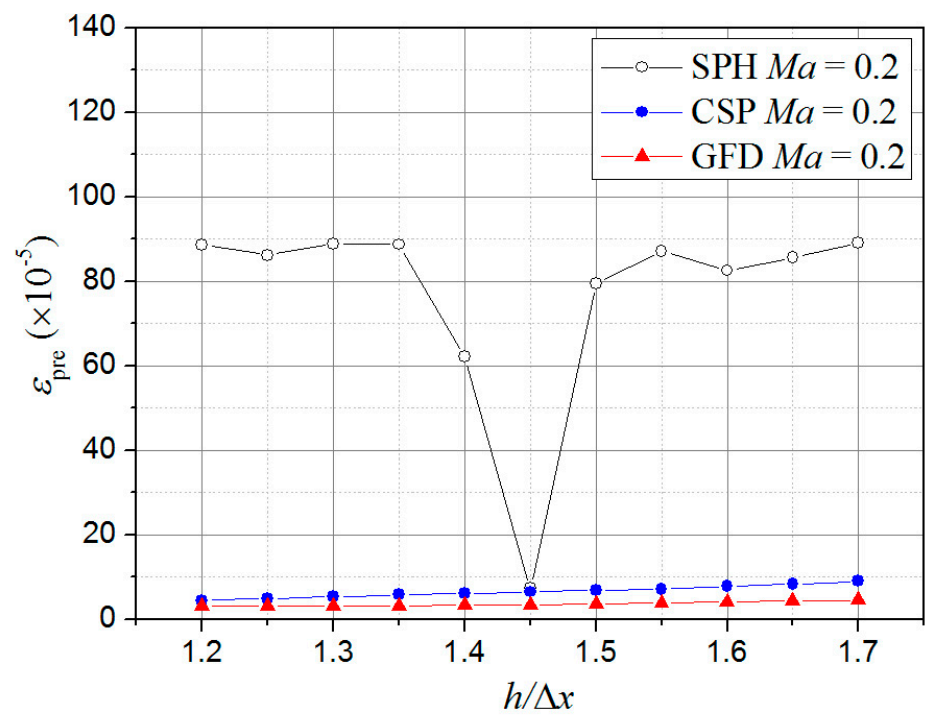

Fig. $8 \varepsilon_{\text {pre }}$ for SPH, CSP, and GFD solutions of LLAPE with different $h$ at $M a=0.2$.

According to the graph, it can be seen that $h / \Delta x$ affects the SPH results a lot, while it has a minimal effect on the accuracy of the CSP and GFD results. For SPH, the minimum $\varepsilon$ pre is obtained when $h / \Delta x$ equals 1.45 . For other cases, Epre rapidly changes to around $8.5 \times 10^{-4}$. On the other hand, the CSP and GFD solutions are accurate and stable, and the GFD method is more accurate than the CSP method. Both results have $\varepsilon$ pre below $1.0 \times 10^{-4}$. Since different cases cost similar $t_{\mathrm{CPU}}$, a comparison of $t_{\mathrm{CPU}}$ of different methods is not given here.

For further evaluation, Table 1 compares the accuracy and efficiency of the three meshfree methods in solving LAPE and LLAPE. Since there is no theoretical solution for LAPE, the exact solutions come from DNS. In the DNS computation, we use a 4th order finite difference scheme for special derivative and a 4th order Runge-Kutta time integration scheme. In order to validate the DNS computation, it is used to solve LLAPE, and the results are compared with theoretical solutions (Eqs. (115)-(117)). The data show that $\varepsilon$ pre of the DNS method is lower than $1.0 \times 10^{-8}$, which is close enough to be considered exact. Considering difference between the DNS mesh and meshfree grids, there is maybe no DNS solution corresponding to a fluid particle in the meshfree computation. The bilinear interpolation is used to interpolate the DNS data to a value corresponding to a fluid particle.

Table 1 Comparison of SPH, CSP, and GFD methods in solving LAPE and LLAPE at different Mach numbers.

\begin{tabular}{cccccccc}
\hline \multirow{2}{*}{ Mach } & & \multicolumn{2}{c}{ SPH } & \multicolumn{2}{c}{ CSP } & \multicolumn{2}{c}{ GFD } \\
Number & Equations & $\varepsilon_{\text {pre }}$ & $t_{\text {CPU }}$ & $\varepsilon_{\text {pre }}$ & $t_{\text {CPU }}$ & $\varepsilon_{\text {pre }}$ & $t_{\text {CPU }}$ \\
& & $\left(\times 10^{-5}\right)$ & $(\mathrm{s})$ & $\left(\times 10^{-5}\right)$ & $(\mathrm{s})$ & $\left(\times 10^{-5}\right)$ & $(\mathrm{s})$ \\
\hline
\end{tabular}




\begin{tabular}{cccccccc}
\hline$M a=-0.2$ & LAPE & 7.2831 & 128 & 6.4546 & 142 & 3.4396 & 143 \\
& LLAPE & 7.6969 & 91 & 6.8671 & 101 & 3.8551 & 119 \\
$M a=0.0$ & LAPE & 7.2828 & 126 & 6.4543 & 142 & 3.4393 & 142 \\
& LLAPE & 7.6965 & 90 & 6.8668 & 102 & 3.8548 & 118 \\
$M a=+0.2$ & LAPE & 7.2824 & 125 & 6.4539 & 143 & 3.4390 & 143 \\
& LLAPE & 7.6962 & 90 & 6.8665 & 102 & 3.8545 & 120 \\
\hline
\end{tabular}

As is shown in the table, both LAPE and LLAPE are validated to be useful in solving problems with acoustic propagation in steady uniform flow. Compared with LAPE, LLAPE can save some computational time with acceptable loss in the accuracy.

The table also indicate that three meshfree methods are successful in numerical implementation of the Lagrangian approach. In solving LAPE, similarly to the previous results of solving LLAPE in Fig. 4 and Fig. 5, the GFD method show a higher accuracy, and the SPH method cost less computational time. In addition, Different Mach numbers of the uniform flow do little effects on $\varepsilon_{\text {pre }}$ and $t_{\text {CPU. }}$.

\subsection{D Sound propagation in unsteady flow}

After analyzing sound propagation in steady uniform flow, the same analysis is used to evaluate the Lagrangian approach in modelling sound propagation in unsteady flow. Theoretical solutions for a 1D unsteady background flow is given in Chapter 3 in [58]. Consider sound propagation in a 1D unsteady flow with

$$
\begin{aligned}
& u_{0}=\frac{2}{\gamma+1} \frac{x}{t}+C_{1} \text {, } \\
& p_{0}=C_{3}\left(\frac{\gamma-1}{C_{3} \gamma}\left(\begin{array}{l}
-\frac{1}{2}\left(C_{1}+\frac{2 x}{(\gamma+1) t}\right)^{2}+\frac{C_{1}^{2}(\gamma+1)}{2(\gamma-1)} \\
+\frac{C_{2}(\gamma-3)}{\gamma+1} t^{\frac{2-2 \gamma}{\gamma+1}}+\frac{x^{2}}{(\gamma+1) t^{2}}
\end{array}\right)\right)^{\gamma /(\gamma-1)}, \\
& \rho_{0}=\left(\frac{(\gamma-1)}{C_{3} \gamma}\left(\begin{array}{l}
-\frac{1}{2}\left(C_{1}+\frac{2 x}{(\gamma+1) t}\right)^{2}+\frac{C_{1}^{2}(\gamma+1)}{2(\gamma-1)} \\
\left.+\frac{C_{2}(\gamma-3)}{\gamma+1} t^{(2-2 \gamma) /(\gamma+1)}+\frac{x^{2}}{(\gamma+1) t^{2}}\right)
\end{array}\right)\right)^{1 /(\gamma-1)} \\
& c_{0}^{2}=\left(\frac{\gamma-1}{\gamma+1} \frac{x}{t}-C_{1}\right)^{2}-\frac{3-\gamma}{\gamma+1}(\gamma-1) C_{2} t^{-2(\gamma-1) /(\gamma+1)},
\end{aligned}
$$


where $u_{0}$ is the initial velocity of background flow, $C_{1}=30.0, C_{2}=-1.0 \times 10^{6}, C_{3}=82571.0$, $\gamma=1.4$, and the initial time $\left(t_{0}\right)$ is at $12.5 \mathrm{~s}$. By selecting these values, the initial velocity of background flow is about $40 \mathrm{~m} / \mathrm{s}$, and the sound speed is around $340 \mathrm{~m} / \mathrm{s}$. Both depend on position.

Sound waves propagate along the $x$-axis over a time interval of $0.5 \mathrm{~s}$, and the initial sound waves are set as Eqs. (112)-(114). The computational domain spans $-250 \mathrm{~m}$ to $250 \mathrm{~m}$. The same Dirichlet boundary conditions to section 5.1 are applied to both ends of the computational region. In the computation, $\Delta x$ is set to be $0.05 \mathrm{~m}, C_{\mathrm{CFL}}$ is 0.15 , and $h$ is selected as $1.45 \Delta x$.

A comparison of pressure obtained from CSP results in solving LLAPE and exact solutions is given in Fig. 9. Fig. 9(b) gives a detailed view of the dashed box in Fig. 9(a). The CSP results are plotted with points at intervals of 30 grid points in figure (a) and 15 grid points in figure (b), and the exact solutions are plotted with solid lines. The exact solutions come from DNS results, and data for the CSP results in the graph is the sum of CSP solutions of sound pressure and $p_{0}$ from Eq. (121). The graph illustrates that CSP results get good agreement with exact solutions in solving LLAPE.

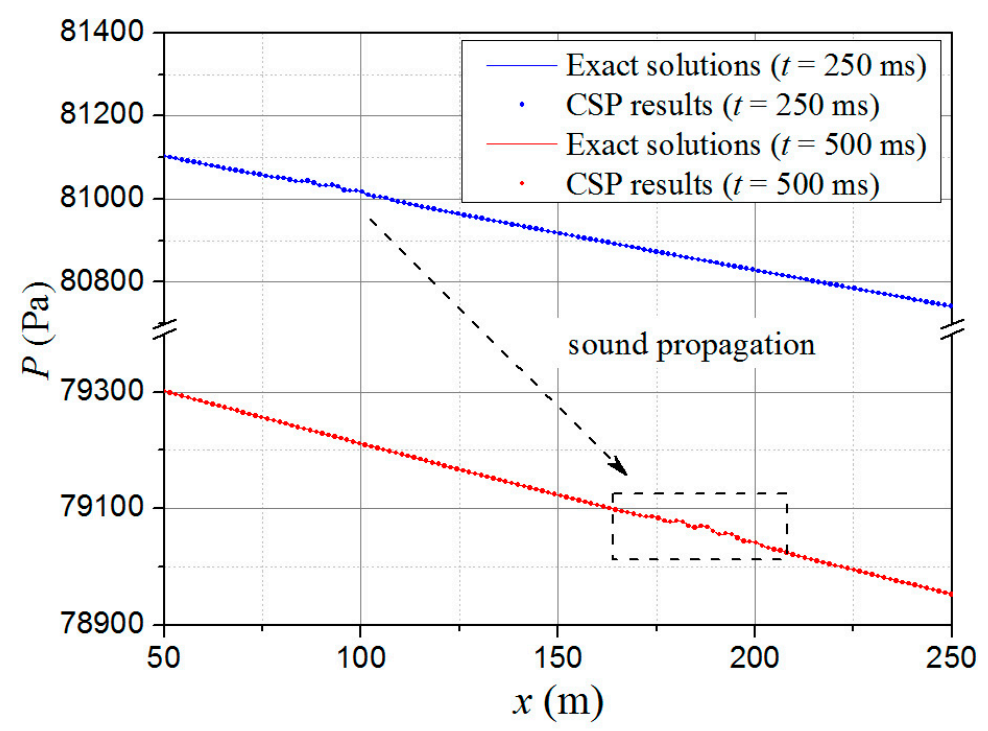

(a) pressure at different times 


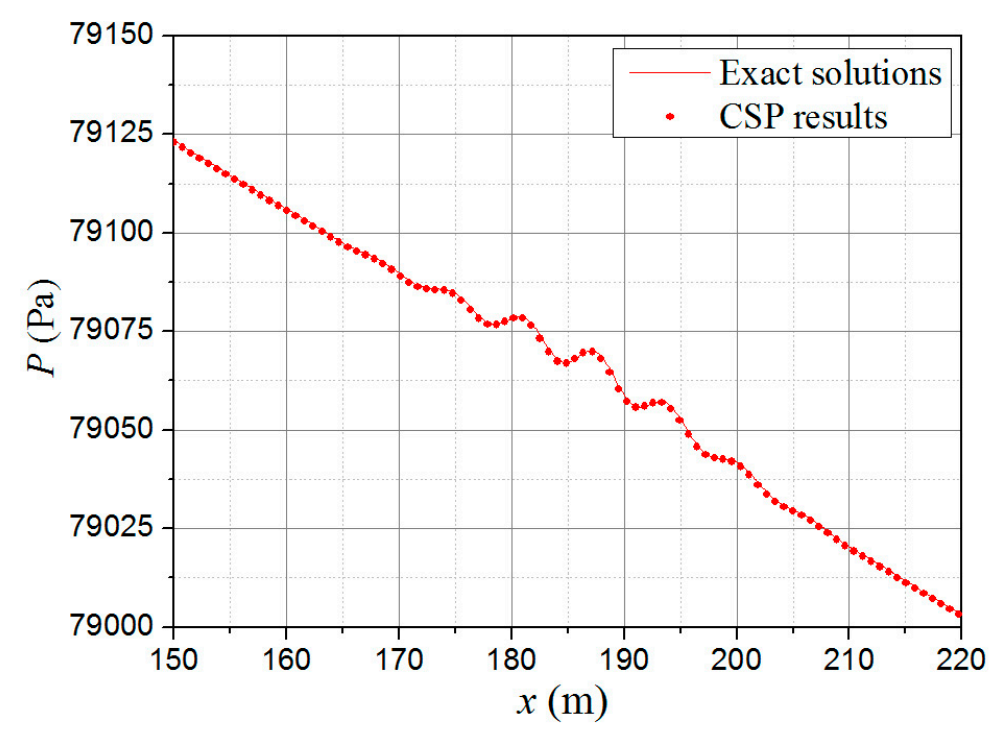

(b) detailed view

Fig. 9 Pressure of CSP solutions of LLAPE versus exact solutions. (a: pressure at different times; b: detailed view of dashed box in (a))

For further evaluation, convergence and CPU time curves for SPH, CSP, and GFD solutions of LLAPE are shown in Fig. 10 and Fig. 11. All computational parameters stay the same as in the model for Fig. 9 except $\Delta x$.

Fig. 10 shows that the SPH method does not converge, while the CSP and GFD methods do. This resultis consistent with the analysis in [59], which gives an error estimation for four schemes including both the standard SPH method and the CSP method. For irregular particle arrangement, [59] shows that the CSP method converges as $\Delta x$ decreases, while convergence for the standard SPH method requires both $\Delta x \rightarrow 0$ and $h / \Delta x \rightarrow \infty$. This conclusion can also be found in [60]. On the other hand, for the CSP and GFD methods, $\varepsilon$ pre decreases along with $\Delta x$. The GFD method has a better accuracy for different $\Delta x$ compare to the other methods. 


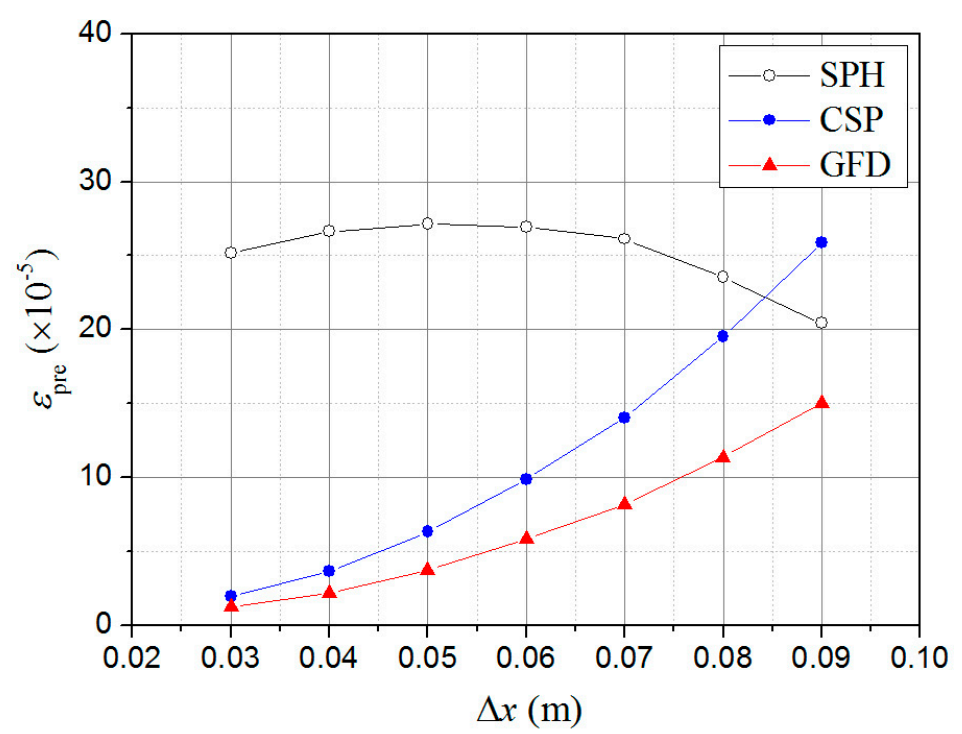

Fig. 10 Convergence curves for SPH, CSP, and GFD solutions of LLAPE.

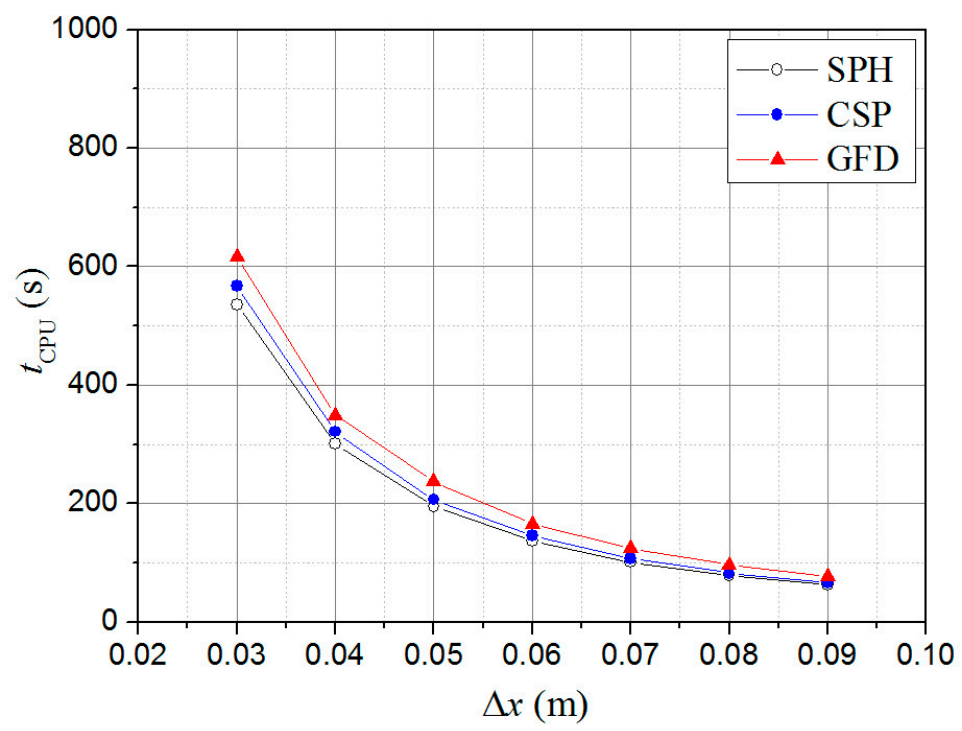

Fig. 11 CPU time curves for SPH, CSP, and GFD solutions of LLAPE at different $\Delta x$.

A decrease of $\Delta x$ increases the time cost as can be seen from Fig. 11. The GFD method has the highest $t_{\mathrm{CPU}}$ cost, while the SPH method has the lowest. Nevertheless, the difference is small between the three methods. $t_{\mathrm{CPU}}$ increases from less than $100 \mathrm{~s}$ to about $600 \mathrm{~s}$ when $\Delta x$ decreases from $0.09 \mathrm{~m}$ to $0.03 \mathrm{~m}$.

To evaluate the effects of $C_{\mathrm{CFL}}$, a comparison of $\varepsilon_{\text {pre from different }} C_{\mathrm{CFL}}$ computation is shown in Fig. 12. When $C_{\text {CFL }}$ is small enough, $\varepsilon_{\text {pre }}$ stays stable for the three methods. When $C_{\mathrm{CFL}}$ increases, $\varepsilon_{\text {pre }}$ diverges. The value of $C_{\mathrm{CFL}}$ to maintain a small and stable $\varepsilon_{\text {pre }}$ for the $\mathrm{SPH}$, CSP, and GFD methods is around $0.23,0.30$, and 0.20 respectively. When $C_{\mathrm{CFL}}$ is smaller than these values, the GFD method is more accurate than other two methods.

A comparison of $t_{\mathrm{CPU}}$ from different $C_{\mathrm{CFL}}$ computation is shown in Fig. 13. Large $C_{\mathrm{CFL}}$ reduces $t_{\mathrm{CPU}}$, and the rate of change slows down as $C_{\mathrm{CFL}}$ increases. The GFD method has the 
highest time cost, but the difference among three methods is small. Increasing $C_{\text {CFL from } 0.05}$ to 0.4 decreases $t_{\mathrm{CPU}}$ from around $600 \mathrm{~s}$ to less than $100 \mathrm{~s}$.

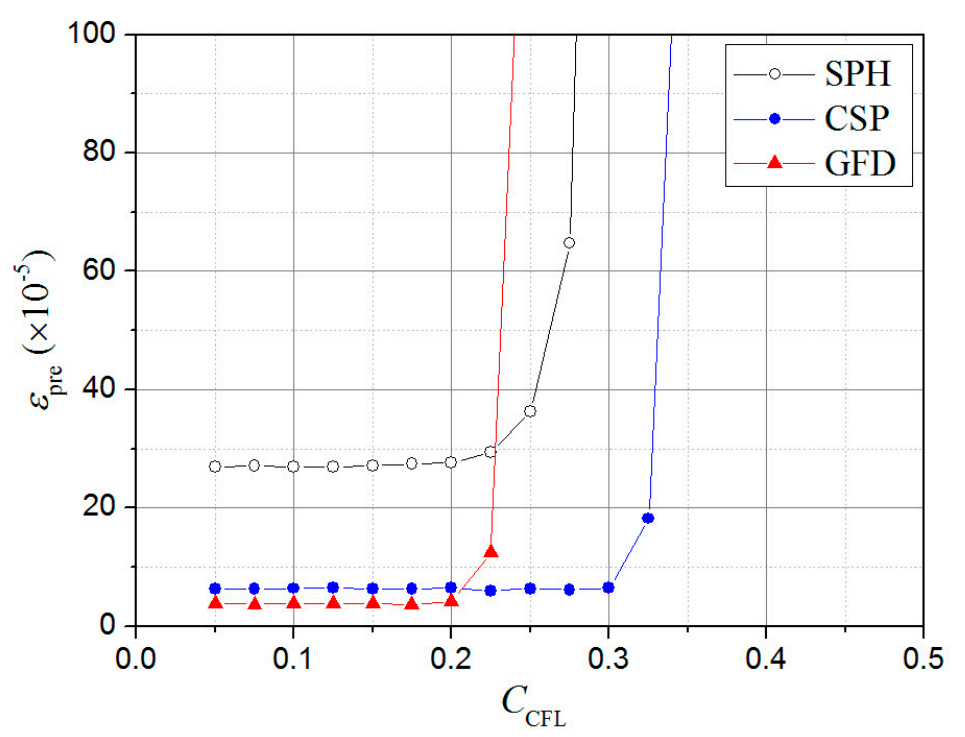

Fig. $12 \varepsilon_{\text {pre }}$ for SPH, CSP, and GFD solutions of LLAPE with different $C_{\mathrm{CFL}}$.

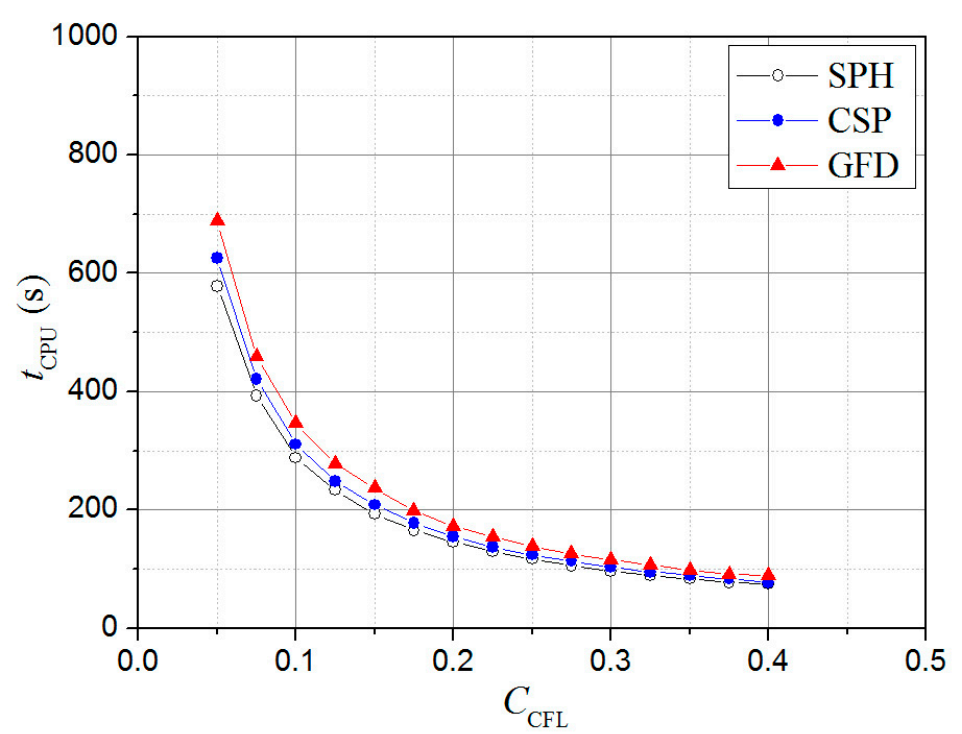

Fig. 13 CPU time curves for SPH, CSP, and GFD solutions of LLAPE with different $C_{\mathrm{CFL}}$.

The effects of $h / \Delta x$ on numerical solutions of LLAPE are shown in Fig. 14. The line graph shows that $\varepsilon$ pre from the SPH method is larger than other two methods, and $h / \Delta x$ affects the SPH results significantly. The most accurate case corresponds to $h / \Delta x$ of 1.45 . The CSP and GFD methods maintain their accuracy well with different $h / \Delta x$. The GFD method is more accurate than the CSP method, but the difference is small. Since a suitable $h$ is unknown before the computation, the CSP and GFD methods appear more practical than the SPH method. 


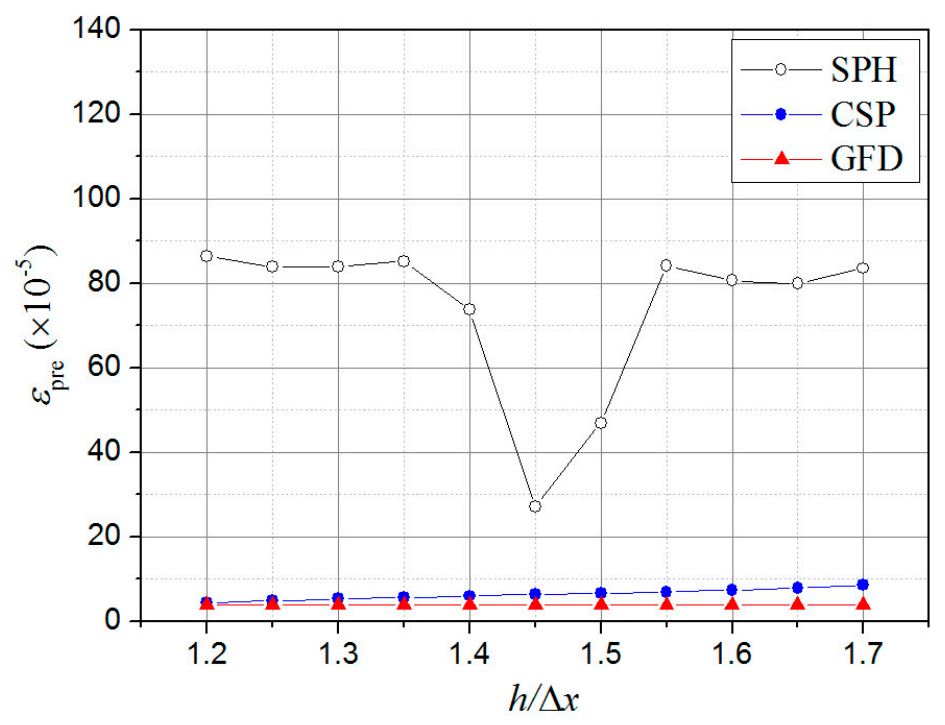

Fig. $14 \varepsilon_{\text {pre }}$ for SPH, CSP, and GFD solutions of LLAPE with different $h$.

For further evaluation, Table 2 compares $\varepsilon$ pre and $t_{\mathrm{CPU}}$ of the SPH, CSP, and GFD methods in solving LAPE and LLAPE with unsteady background flow.

Table 2 Comparison of SPH, CSP, and GFD methods in solving LAPE and LLAPE with unsteady flow.

\begin{tabular}{ccccccc}
\hline & \multicolumn{2}{c}{ SPH } & \multicolumn{2}{c}{ CSP } & \multicolumn{2}{c}{ GFD } \\
Equations & $\varepsilon_{\text {pre }}$ & $t_{\text {CPU }}$ & $\varepsilon_{\text {pre }}$ & $t$ CPU & $\varepsilon_{\text {pre }}$ & $t_{\text {CPU }}$ \\
& $\left(\times 10^{-5}\right)$ & $(\mathrm{s})$ & $\left(\times 10^{-5}\right)$ & $(\mathrm{s})$ & $\left(\times 10^{-5}\right)$ & $(\mathrm{s})$ \\
\hline LAPE & 27.1432 & 267 & 5.8515 & 284 & 3.2900 & 279 \\
LLAPE & 27.5736 & 195 & 6.3053 & 207 & 3.7422 & 237 \\
\hline
\end{tabular}

The table indicates that both LAPE and LLAPE are able to solve acoustic problems with unsteady background flow. In addition, LLAPE save much computational time with acceptable loss in the accuracy comparing with LAPE. In solving LAPE, similarly to the previous results of solving LLAPE in Fig. 10 and Fig. 11, the GFD method is more accurate than the other two methods, while the SPH method cost the least computational time.

\subsection{D sound propagation in steady uniform flow}

In this case, a 2D acoustic model is selected to validate the Lagrangian approach. This model is similar to the problem given in the ICASE/LaRC workshop concerning benchmark problems in computational aeroacoustics (CAA) [61]. In addition, the standard SPH method is not discussed in 2D problems, because it has more limitation to maintain convergence and the selection of $h$ is also more difficult than the CSP and GFD methods in the application as shown in Section 5.1 and 5.2.

Consider sound propagation in steady uniform flow with $M a=0.2$. Let $\alpha_{1}=10 / 340^{2}$, 
$\alpha_{2}=\ln 2 / 9, \alpha_{3}=\ln 2 / 7, \eta=\sqrt{\left(x^{2}+y^{2}\right)}$, where $x$ and $y$ are the position coordinates. The initial conditions are

$$
\begin{gathered}
\delta p=\alpha_{1} \rho_{0} c_{0}^{2} \exp \left[-\alpha_{2}\left(x^{2}+y^{2}\right)\right] \\
\delta \rho=\alpha_{1} \exp \left[-\alpha_{2}\left(x^{2}+y^{2}\right)\right]+0.1 \alpha_{1} \exp \left[-\alpha_{3}\left((x-10)^{2}+y^{2}\right)\right] \\
\delta u=0.04 \alpha_{1} c_{0} y \exp \left[-\alpha_{3}\left((x-10)^{2}+y^{2}\right)\right] \\
\delta v=-0.04 \alpha_{1} c_{0}(x-10) \exp \left[-\alpha_{3}\left((x-10)^{2}+y^{2}\right)\right]
\end{gathered}
$$

where $\delta u$ and $\delta v$ are the velocity perturbation of particles along the $x$-axis and $y$-axis respectively. The fluid is an ideal gas with sound speed $c_{0} 340 \mathrm{~m} / \mathrm{s}$ and $\rho_{0} 1.0 \mathrm{~kg} / \mathrm{m}^{3}$. The exact solutions for the sound waves when time equals $t$ are

$$
\begin{gathered}
\delta p=\frac{\alpha_{1} \rho_{0} c_{0}^{2}}{2 \alpha_{2}} \int_{0}^{\infty} \exp \left(\frac{-\xi^{2}}{4 \alpha_{2}}\right) \cos (\xi t) J_{0}(\xi \eta) \xi d \xi \\
\delta \rho=\frac{\alpha_{1}}{2 \alpha_{2}} \int_{0}^{\infty} \exp \left(\frac{-\xi^{2}}{4 \alpha_{2}}\right) \cos (\xi t) J_{0}(\xi \eta) \xi d \xi \\
+0.1 \alpha_{1} \exp \left[-\alpha_{3}\left((x-10)^{2}+y^{2}\right)\right] \\
\delta u=\frac{\alpha_{1} c_{0} x}{2 \alpha_{2} \eta} \int_{0}^{\infty} \exp \left(\frac{-\xi^{2}}{4 \alpha_{2}}\right) \sin (\xi t) J_{1}(\xi \eta) \xi d \xi \\
+0.04 \alpha_{1} c_{0} y \exp \left[-\alpha_{3}\left((x-10)^{2}+y^{2}\right)\right] \\
\delta v=\frac{\alpha_{1} c_{0} y}{2 \alpha_{2} \eta} \int_{0}^{\infty} \exp \left(\frac{-\xi^{2}}{4 \alpha_{2}}\right) \sin \left(\xi_{t}\right) J_{1}(\xi \eta) \xi d \xi \\
-0.04 \alpha_{1} c_{0}(x-10) \exp \left[-\alpha_{3}\left((x-10)^{2}+y^{2}\right)\right]
\end{gathered}
$$

where $J_{0}()$ and $J_{1}($ ) are Bessel functions of order 0 and 1 respectively.

In the simulation, $\Delta x$ is set as $0.2 \mathrm{~m}, C_{\mathrm{CFL}}$ is selected as 0.2 , and $h$ is $1.1 \Delta x$. The boundary particles around the computational domain are taken to be Dirichlet boundary with perturbation variables set to 0 . A model with larger computational domain is implemented to show the process of sound propagation in the uniform flow at first, then a smaller model is used for the evaluation in order to save the time.

The CSP results for the change in density at different time steps are shown in Fig. 15. In this simulation, the computational domain is $-80 \mathrm{~m}<\mathrm{x}<80 \mathrm{~m},-80 \mathrm{~m}<\mathrm{y}<80 \mathrm{~m}$. During 
the computation, all computational particles are moving along with the background flow, and sound waves propagate in the media at the same time. The direction of the background flow is represented by an arrow. Computational results show that the CSP method can simulate the propagation process of Gaussian impulses.

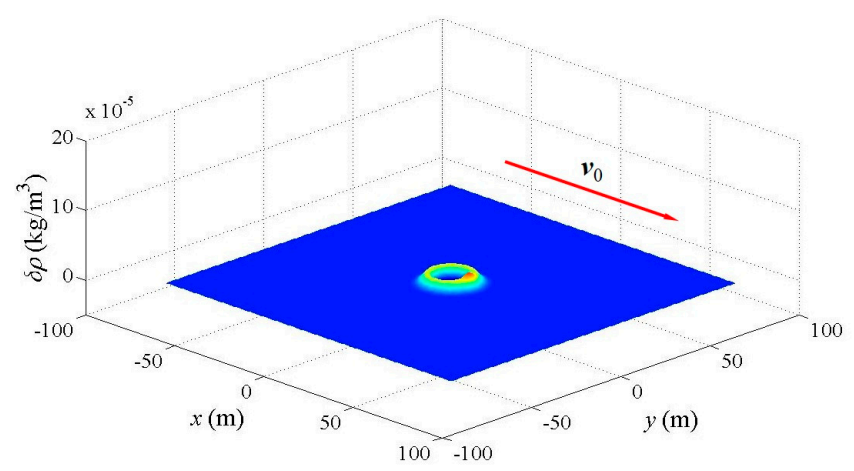

(a) $0.025 \mathrm{~s}$

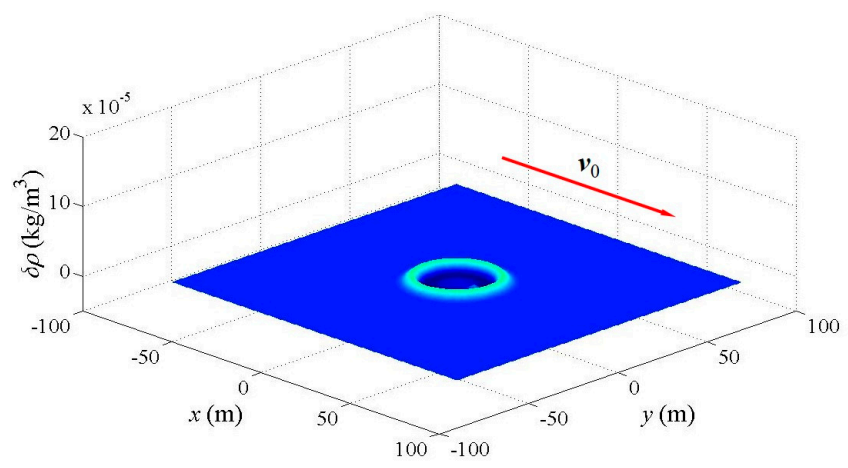

(b) $0.05 \mathrm{~s}$

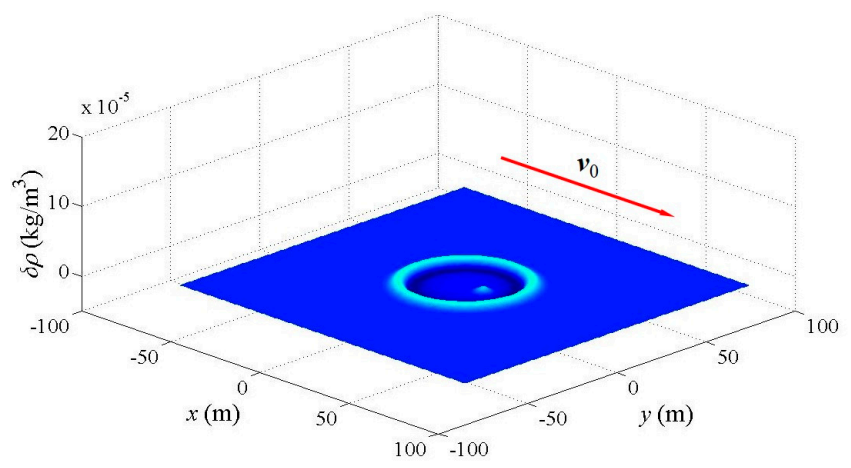

(c) $0.075 \mathrm{~s}$ 


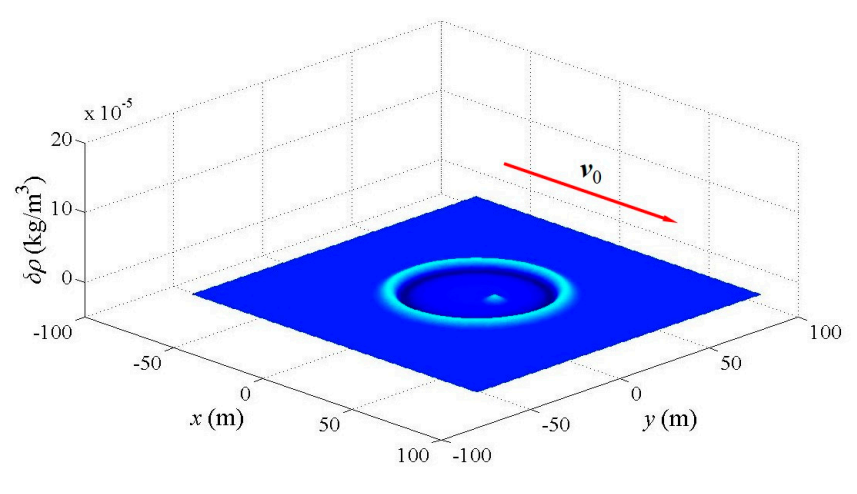

(d) $0.10 \mathrm{~s}$

Fig. 15 Contour of density change obtained by the CSP method at different time.

To evaluate the computational results, Fig. 16 gives a comparison between CSP results and DNS solutions along the $x$-axis. In this simulation, the computational domain is $-40 \mathrm{~m}<$ $\mathrm{x}<40 \mathrm{~m},-40 \mathrm{~m}<\mathrm{y}<40 \mathrm{~m}$, and the time for sound propagation is $50 \mathrm{~ms}$. The points in the figure represent the numerical values at different positions, and the lines denote the exact solutions. In order to clearly identify numerical results, the points are plotted at intervals of 5 grid points. Since the GFD results are similar to Fig. 16, they are not shown in here. As can be seen from the line graph, numerical results of all perturbation variables agrees well with exact solutions. The comparison suggests that the CSP results are accurate.

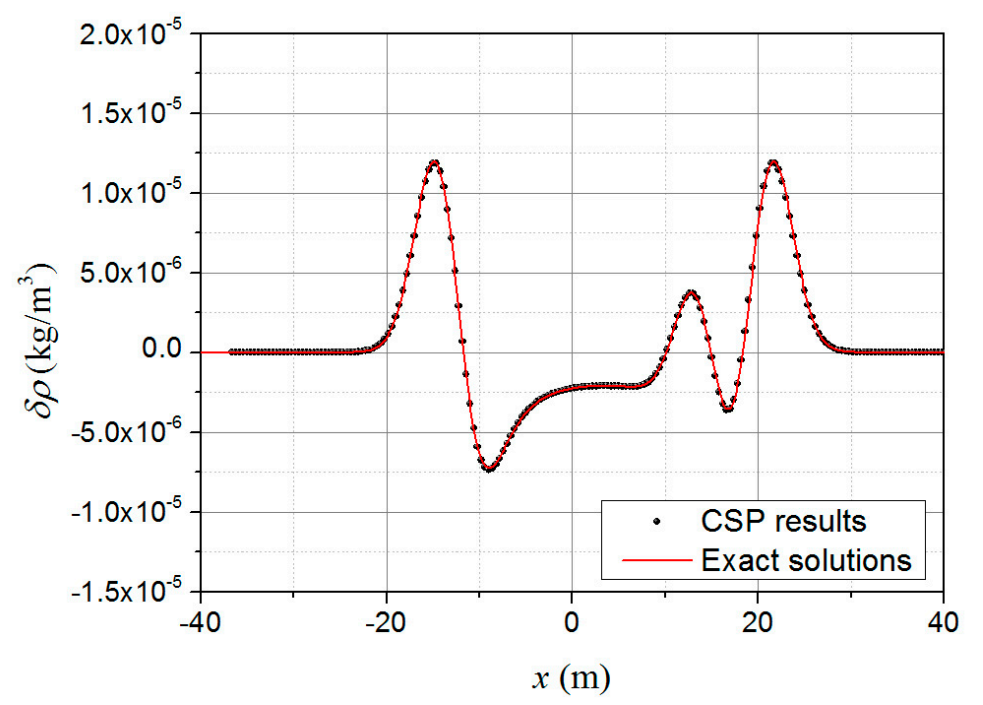

(a) density change 


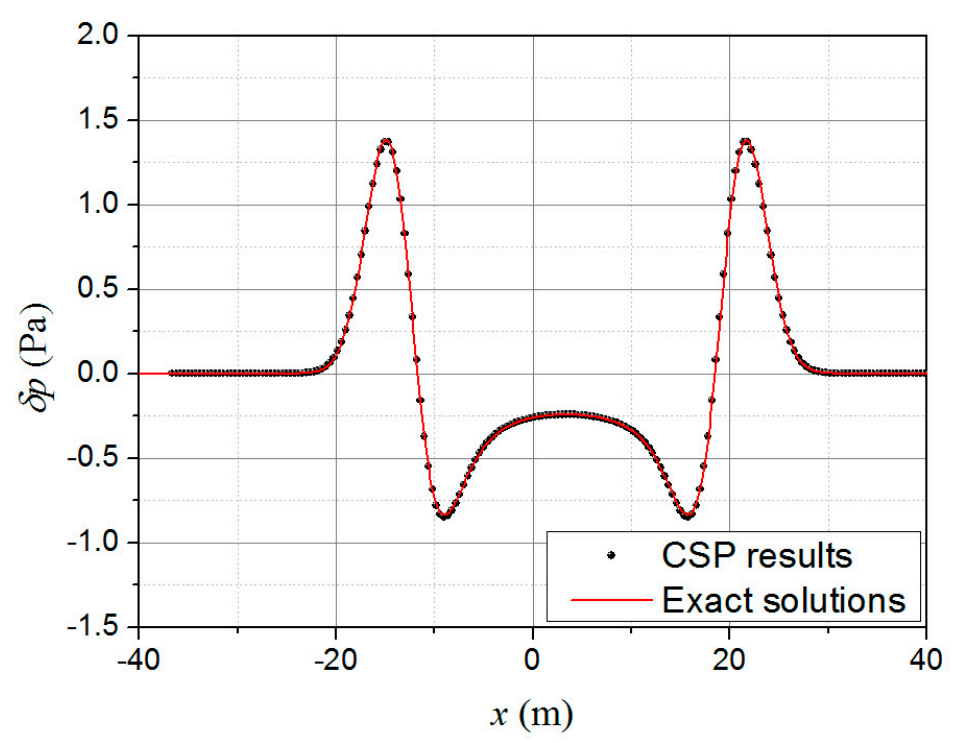

(b) sound pressure

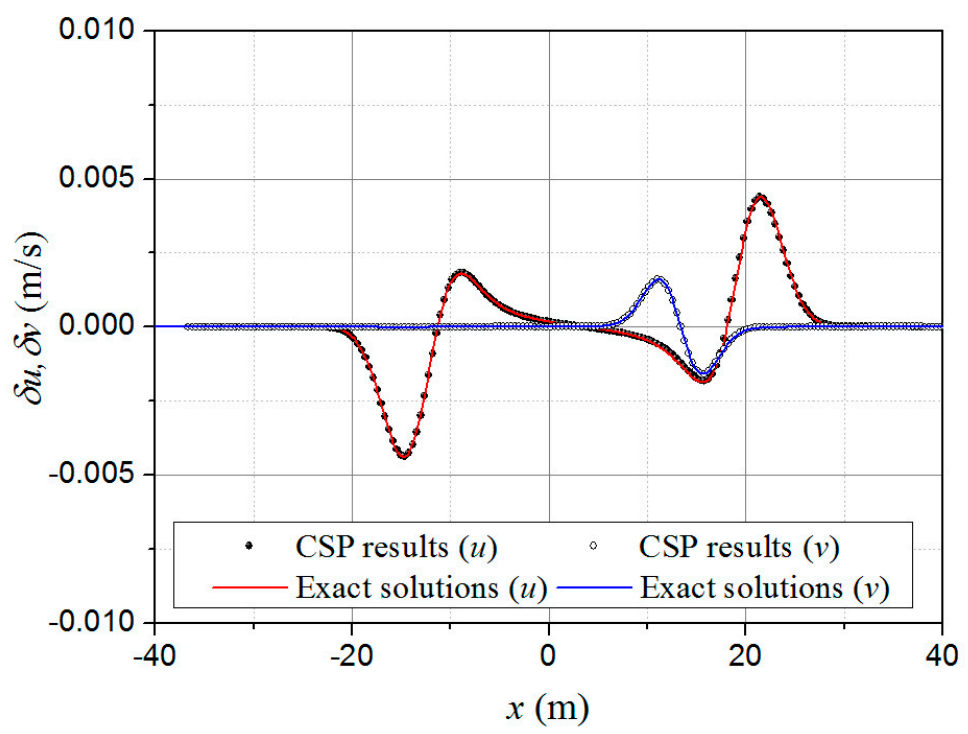

(c) velocity perturbation

Fig. 16 Comparison between CSP results and exact solutions along the $x$-axis at $0.05 \mathrm{~s}$.

Convergence and CPU time curves for CSP and GFD solutions of LLAPE are shown in Fig. 17 and Fig. 18. As can be seen from the figure, both the CSP and GFD methods converge when solving LLAPE. The GFD method has better accuracy for different $\Delta x$ compared to the CSP method. $\varepsilon$ pre for the two meshfree methods increases from about $2.0 \times 10^{-6}$ to more than $1.0 \times 10^{-4}$ along with $\Delta x$ increasing from 0.1 to $0.4 \mathrm{~m}$. However, $t_{\mathrm{CPU}}$ decreases significantly along as $\Delta x$ increase, and the GFD method costs more $t_{\mathrm{CPU}}$ than the CSP method. For the two methods, $t_{\mathrm{CPU}}$ changes from more than $1000 \mathrm{~s}$ to around $20 \mathrm{~s}$ when $\Delta x$ changes from $0.10 \mathrm{~m}$ to $0.40 \mathrm{~m}$. 


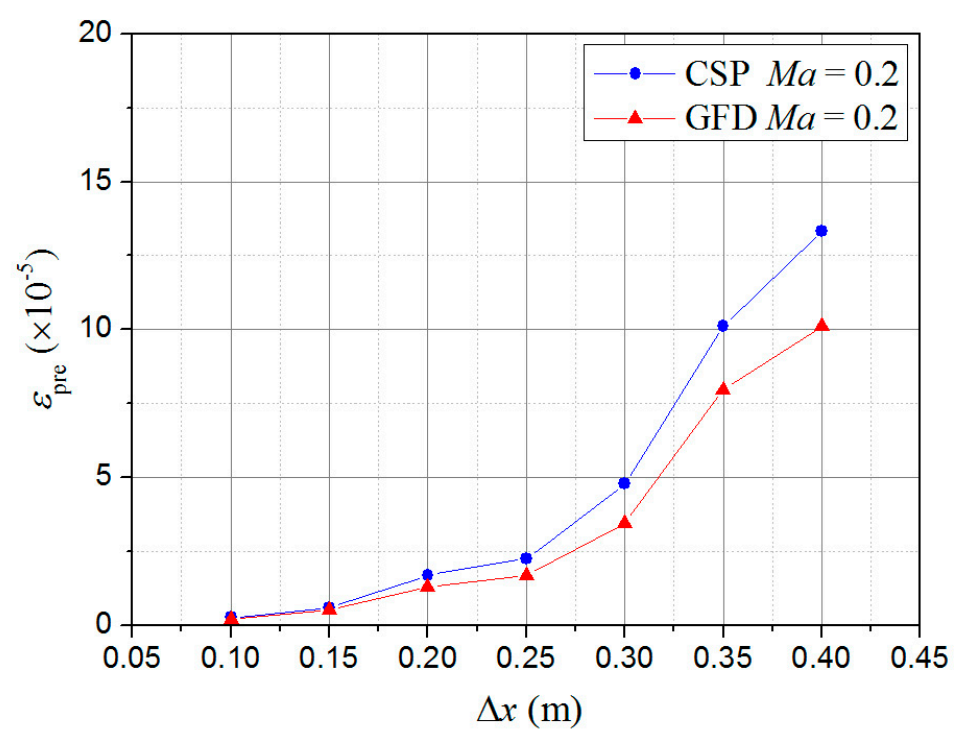

Fig. 17 Convergence curves for CSP and GFD solutions of LLAPE at Ma=0.2.

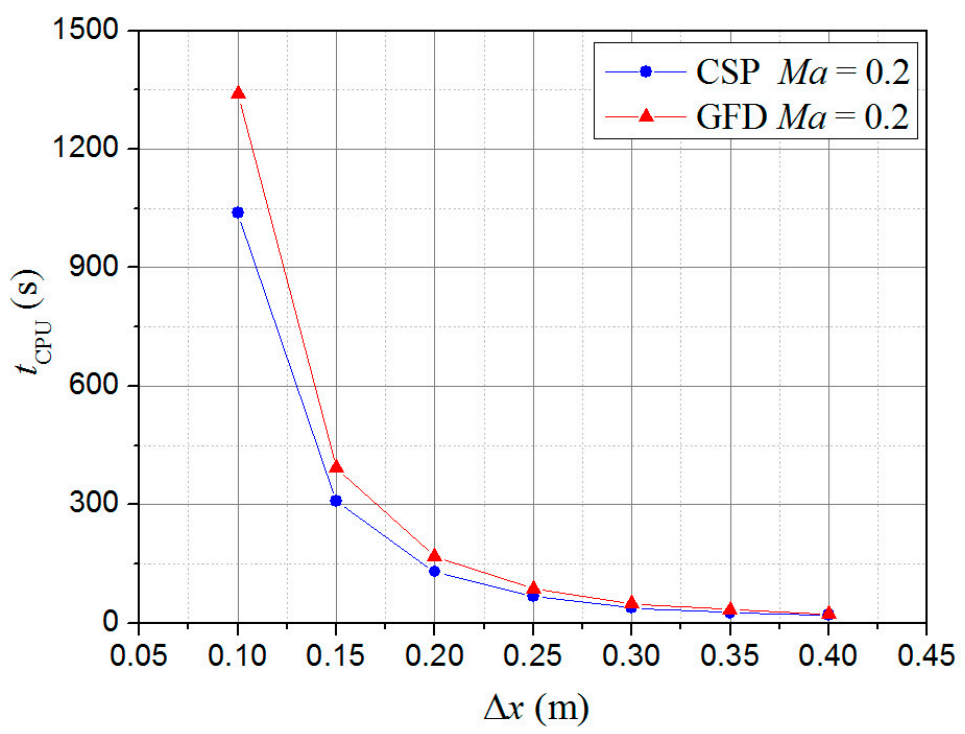

Fig. $18 \mathrm{CPU}$ time curves for CSP and GFD solutions of LLAPE at $M a=0.2$ at different $\Delta x$.

The effects of $C_{\mathrm{CFL}}$ on the CSP and GFD solutions of LLAPE at $M a=0.2$ are shown in Fig. 19 and Fig. 20. Fig. 19 indicates that an appropriate $C_{\mathrm{CFL}}$ is necessary for accurate numerical results. When $C_{\mathrm{CFL}}$ is small enough, $\varepsilon$ pre stays low value and the method remains stable. An increase of $C_{\mathrm{CFL}}$ leads to divergence in $\varepsilon$ pre. The methods remain stable when $C_{\mathrm{CFL}}$ $<0.2$. After that, $\varepsilon$ pre fluctuates slightly but still acceptable since $\varepsilon$ pre is under $5.0 \times 10^{-5}$. Finally, $\varepsilon$ pre rises sharply when $C_{\mathrm{CFL}}$ is larger than 0.7 and 0.55 for the CSP and GFD method respectively. For most acceptable choices of $C_{\mathrm{CFL}}$, the GFD method has lower $\varepsilon_{\text {pre }}$ than the CSP method. 


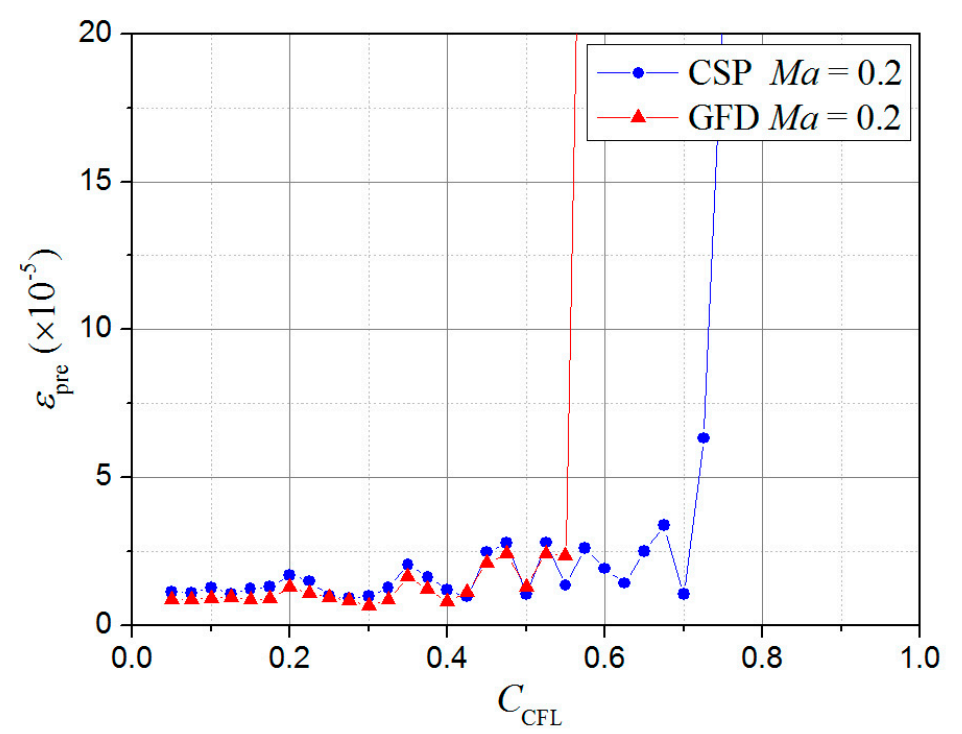

Fig. $19 \varepsilon_{\text {pre }}$ for CSP and GFD solutions of LLAPE at $M a=0.2$ with different $C_{\mathrm{CFL}}$.

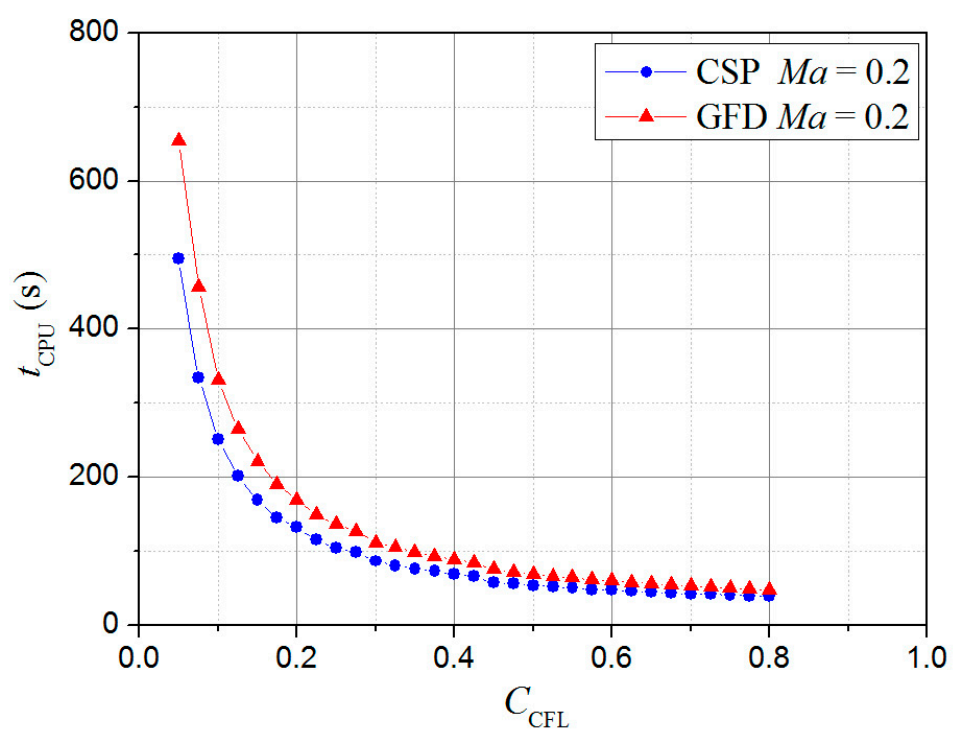

Fig. 20 CPU time curves for CSP and GFD solutions of LLAPE at $M a=0.2$ with different $C_{\mathrm{CFL}}$.

Fig. 20 shows that for small $C_{\mathrm{CFL}}, t_{\mathrm{CPU}}$ grows rapidly, and the GFD method costs more than the CSP method. For the CSP method, $C_{\mathrm{CFL}}$ changes from 0.05 to 0.8 decreases $t$ CPU from about $500 \mathrm{~s}$ to $50 \mathrm{~s}$.

Table 3 compares the accuracy and efficiency of the CSP and GFD methods when solving LAPE and LLAPE.

Table 3 Comparison of CSP and GFD methods in solving LAPE and LLAPE with uniform flow.

\begin{tabular}{ccccc}
\hline & \multicolumn{2}{c}{ CSP } & \multicolumn{2}{c}{ GFD } \\
Equations & $\varepsilon_{\text {pre }}$ & $t_{\mathrm{CPU}}$ & $\varepsilon_{\text {pre }}$ & $t_{\mathrm{CPU}}$ \\
& $\left(\times 10^{-5}\right)$ & $(\mathrm{s})$ & $\left(\times 10^{-5}\right)$ & $(\mathrm{s})$ \\
\hline LAPE & 1.6742 & 221 & 1.2672 & 190 \\
LLAPE & 1.6805 & 130 & 1.2735 & 169 \\
\hline & & 36
\end{tabular}


The table shows that both LAPE and LLAPE are able to solve 2D acoustic problems in steady uniform flow, and LLAPE can save some computational time with acceptable loss in the accuracy comparing with LAPE. Moreover, the GFD method shows more accurate than the CSP method.

\section{$5.42 \mathrm{D}$ sound propagation in rotational vortex}

$2 \mathrm{D}$ sound propagation in a rotational vortex is considered in this case. The vortex is described by

$$
\begin{gathered}
u_{0}=-\omega y, \\
v_{0}=\omega x, \\
p_{0}=\frac{1}{2} \omega^{2} x^{2}+\frac{1}{2} \omega^{2} y^{2}+C_{\mathrm{pre}},
\end{gathered}
$$

where $\omega$ is the angular velocity of the rotational vortex, $x$ and $y$ are the position coordinates, and $u 0$ and $v_{0}$ are the velocity of particles along the $x$-axis and $y$-axis respectively.

A $2 \mathrm{D}$ acoustic model similar to section 5.3 is selected to validate the Lagrangian approach. Let $\quad \alpha_{1}=\frac{10}{340^{2}}, \alpha_{2}=\frac{\ln 2}{9}, \alpha_{3}=\frac{\ln 2}{7}$.

The initial conditions are

$$
\begin{gathered}
\delta p=\alpha_{1} \rho_{0} c_{0}^{2} \exp \left\{-\alpha_{2}\left[(x-10)^{2}+(y-10)^{2}\right]\right\} \\
\delta \rho=\alpha_{1} \exp \left\{-\alpha_{2}\left[(x-10)^{2}+(y-10)^{2}\right]\right\} \\
+0.1 \alpha_{1} \exp \left\{-\alpha_{3}\left[(x-20)^{2}+(y-10)^{2}\right]\right\} \\
\delta u=0.04 \alpha_{1} c_{0}(y-10) \exp \left\{-\alpha_{3}\left[(x-20)^{2}+(y-10)^{2}\right]\right\} \\
\delta v=-0.04 \alpha_{1} c_{0}(x-20) \exp \left\{-\alpha_{3}\left[(x-20)^{2}+(y-10)^{2}\right]\right\}
\end{gathered}
$$

A model with larger computational domain is implemented to show the process of sound propagation in the rotational flow at first, then a smaller model is used for the evaluation in order to save the time.

The contour of density change at different time is shown in Fig. 21. In this simulation, the computational domain is $-30 \mathrm{~m}<x<130 \mathrm{~m},-30 \mathrm{~m}<y<130 \mathrm{~m}$, and $\omega$ is set as $8 \mathrm{rad} / \mathrm{s}$. As shown in the contour, the red line at position $(20,10)$ is the center of the rotational vortex. All particles are moving during the computation, and sound waves propagate in the media at the same time. 


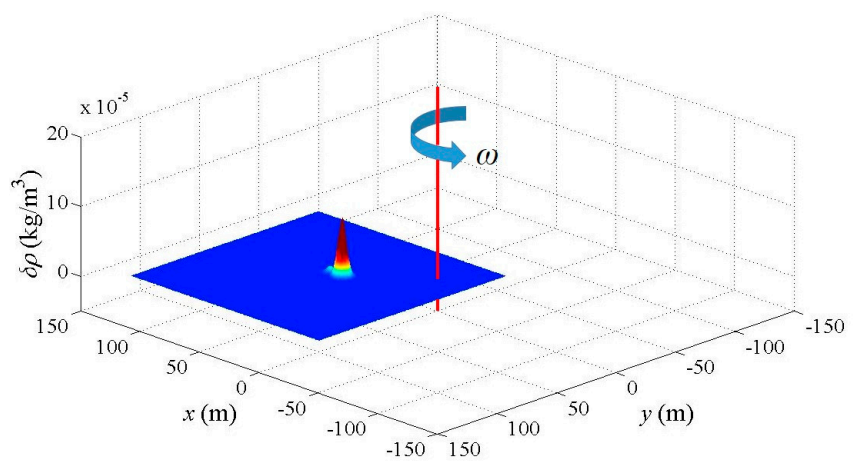

(a) $0 \mathrm{~s}$

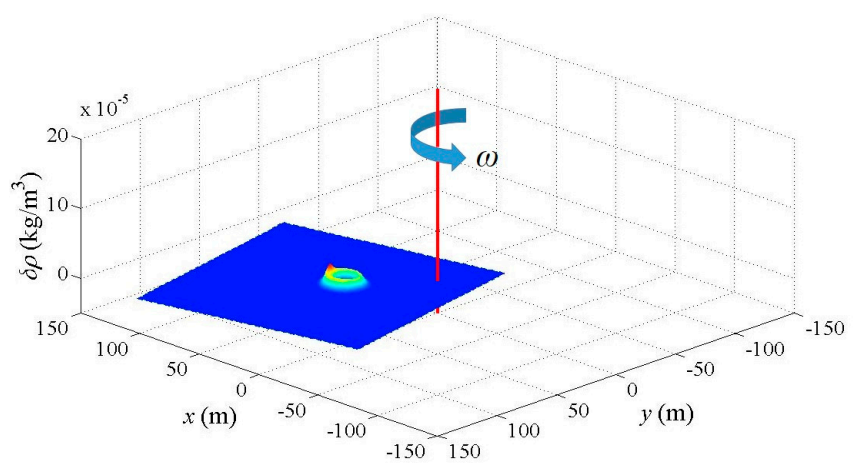

(b) $0.025 \mathrm{~s}$

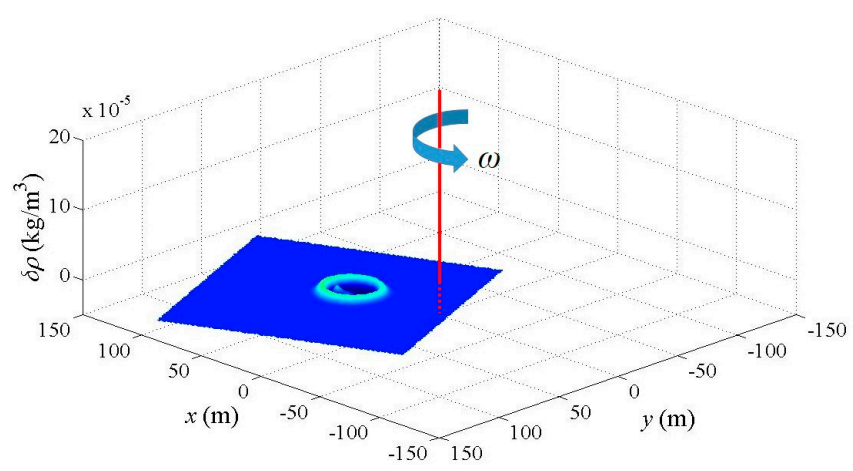

(c) $0.05 \mathrm{~s}$

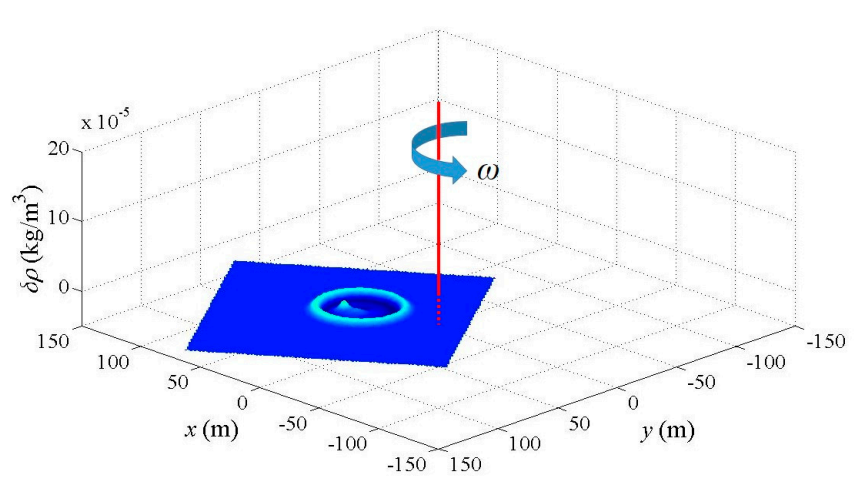




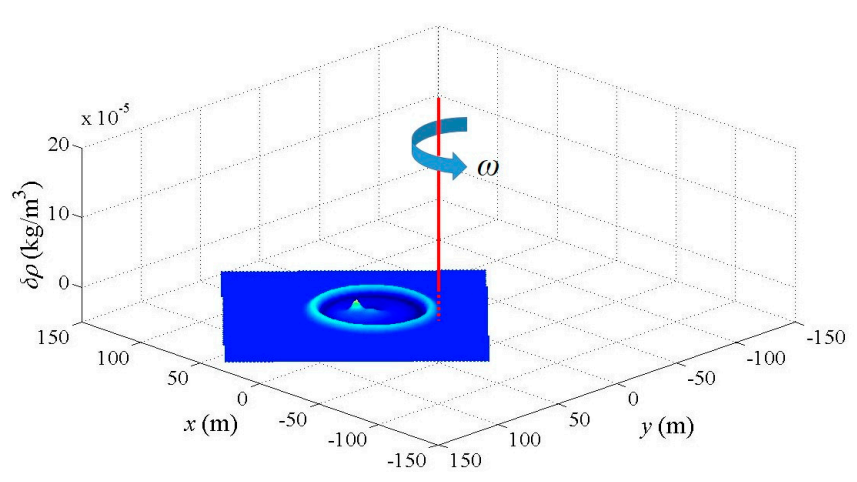

(c) $0.10 \mathrm{~s}$

Fig. 21 Contour of CSP solutions of density change at different time.

For further evaluation, a model close to the actual situation is considered. The computational domain is $-30 \mathrm{~m}<x<50 \mathrm{~m},-30 \mathrm{~m}<y<50 \mathrm{~m}$, and $\omega$ is set as $2 \mathrm{rad} / \mathrm{s}$. Fig. 22 gives a comparison of density change between the CSP method and exact solutions at $y=10$ $\mathrm{m}$. The line represents exact solutions, and points in the figure represent the CSP results. As can be seen from the line graph, the CSP results show good agreement with the exact solutions.

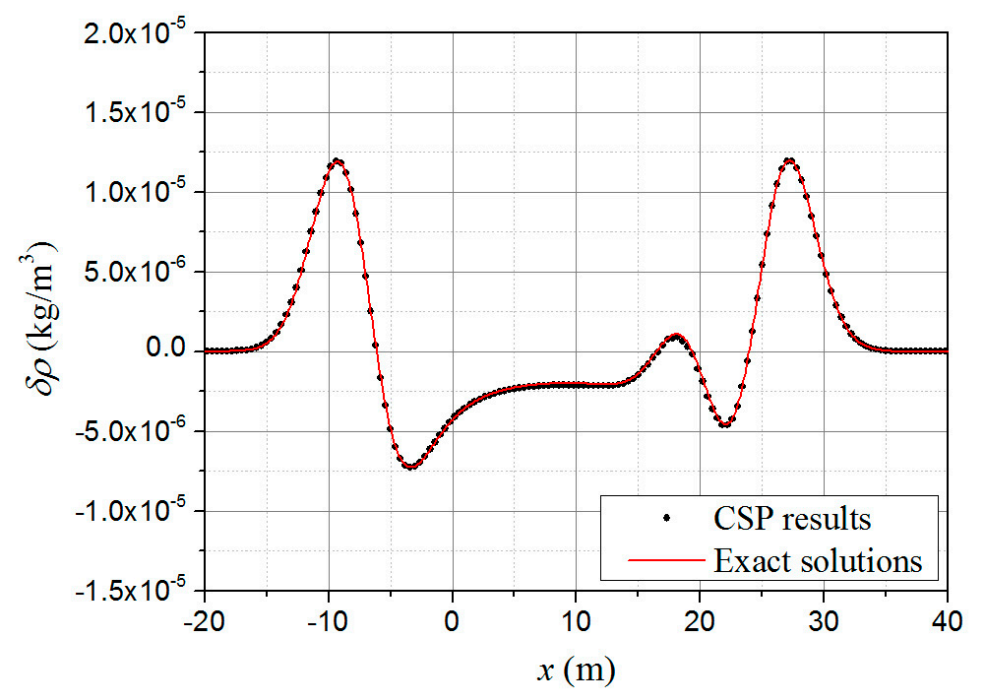

Fig. 22 Comparison of density change between CSP results and exact solutions along $y=10 \mathrm{~m}$.

Convergence and CPU time curves for CSP and GFD solutions of LLAPE are shown in Fig. 23 and Fig. 24. All computational parameters stay the same as in section 5.3 except $\Delta x$.

Fig. 23 shows that both the CSP and GFD method are convergent in this case, and the GFD method has better accuracy for different $\Delta x$ compared to the CSP method. $\varepsilon$ pre for two meshfree methods increases from less than $1.0 \times 10^{-5}$ to over $1.0 \times 10^{-4}$ along with $\Delta x$ changing from 0.1 to $0.4 \mathrm{~m}$. However, a decrease of $\Delta x$ increases the time cost as can be seen from Fig. 24. The GFD method has a higher time cost than the CSP method. For both 
meshfree methods, $t_{\mathrm{CPU}}$ increases over 10 times when $\Delta x$ decreases from 0.4 to $0.1 \mathrm{~m}$.

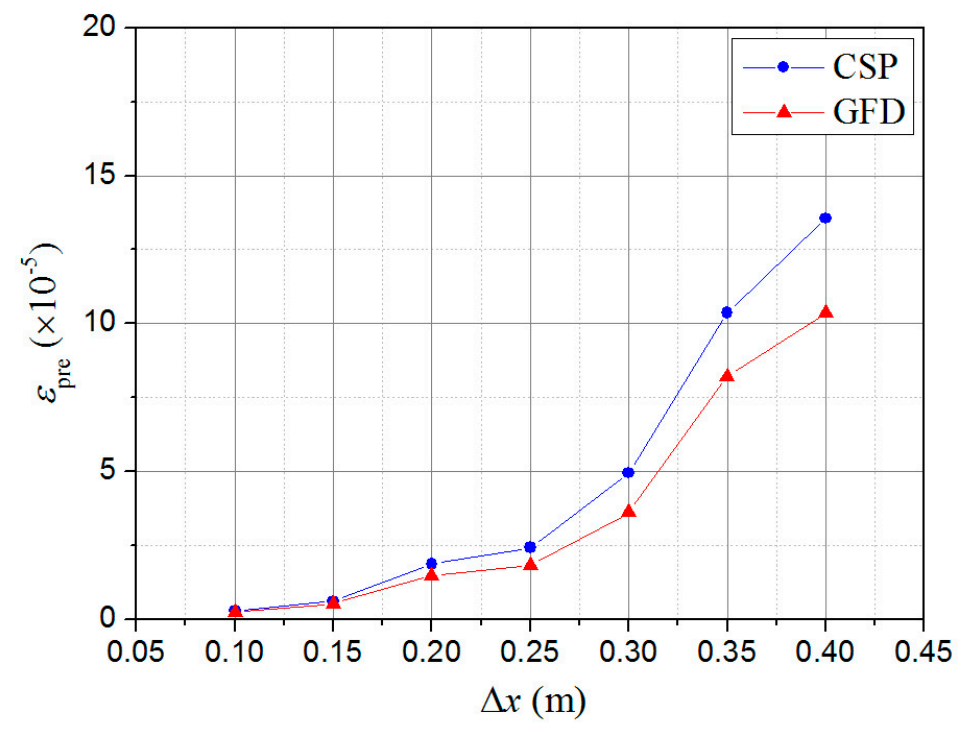

Fig. 23 Convergence curves for CSP and GFD solutions of LLAPE.

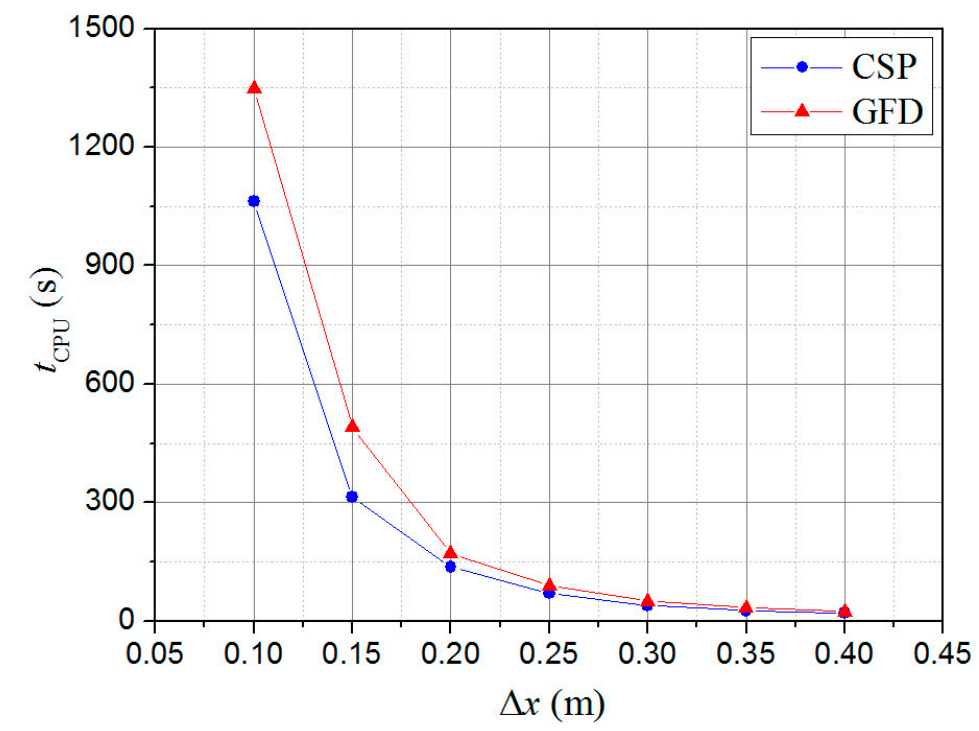

Fig. 24 CPU time curves for CSP and GFD solutions of LLAPE at different $\Delta x$.

To evaluate the effects of $C_{\mathrm{CFL}}$, a comparison of $\varepsilon_{\text {pre }}$ from the computation with different

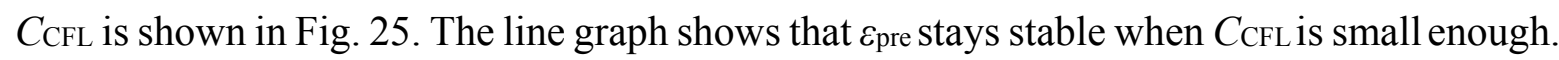
There is then a clear increase in $\varepsilon$ pre when $C_{\text {CFL }}$ increases and gets higher. The stability condition for $C_{\mathrm{CFL}}$ is different for the two methods. According to the graph, the highest $C_{\mathrm{CFL}}$ value for the CSP and GFD methods is about 0.7 and 0.5 separately.

A comparison of $t_{\mathrm{CPU}}$ from different $C_{\mathrm{CFL}}$ computations is shown in Fig. 26. The figure shows the GFD method has a higher $t_{\mathrm{CPU}}$ compared to the CSP method. For the two methods, changing $C_{\mathrm{CFL}}$ from 0.05 to 0.8 decreases $t_{\mathrm{CPU}}$ from over $500 \mathrm{~s}$ to about $50 \mathrm{~s}$. 


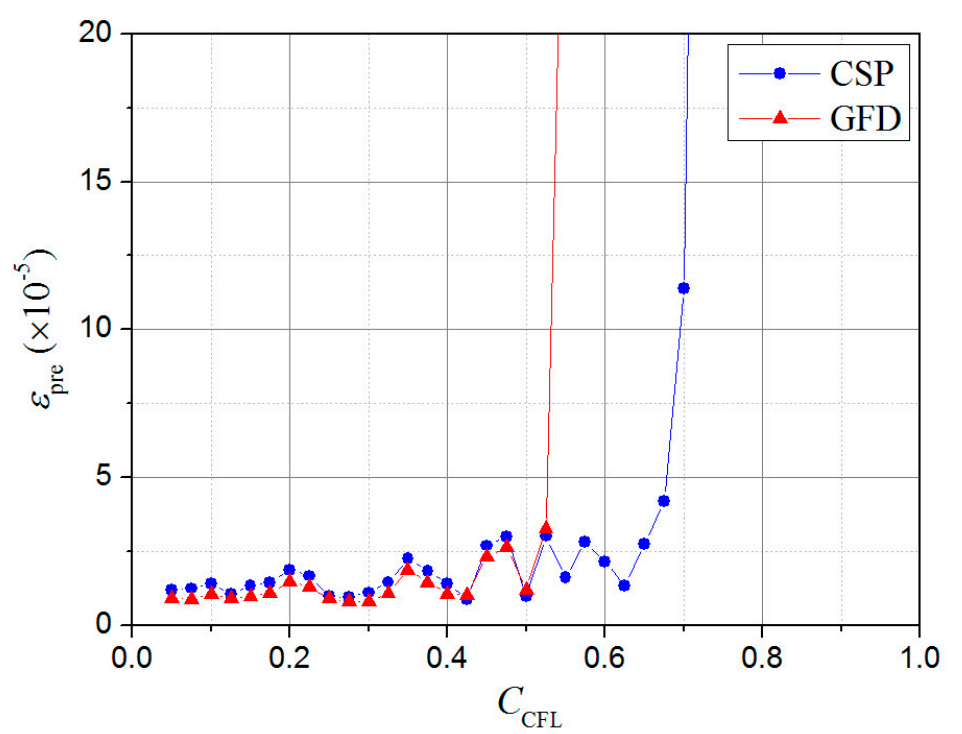

Fig. $25 \varepsilon_{\text {pre }}$ for CSP and GFD solutions of LLAPE with different CFL numbers.

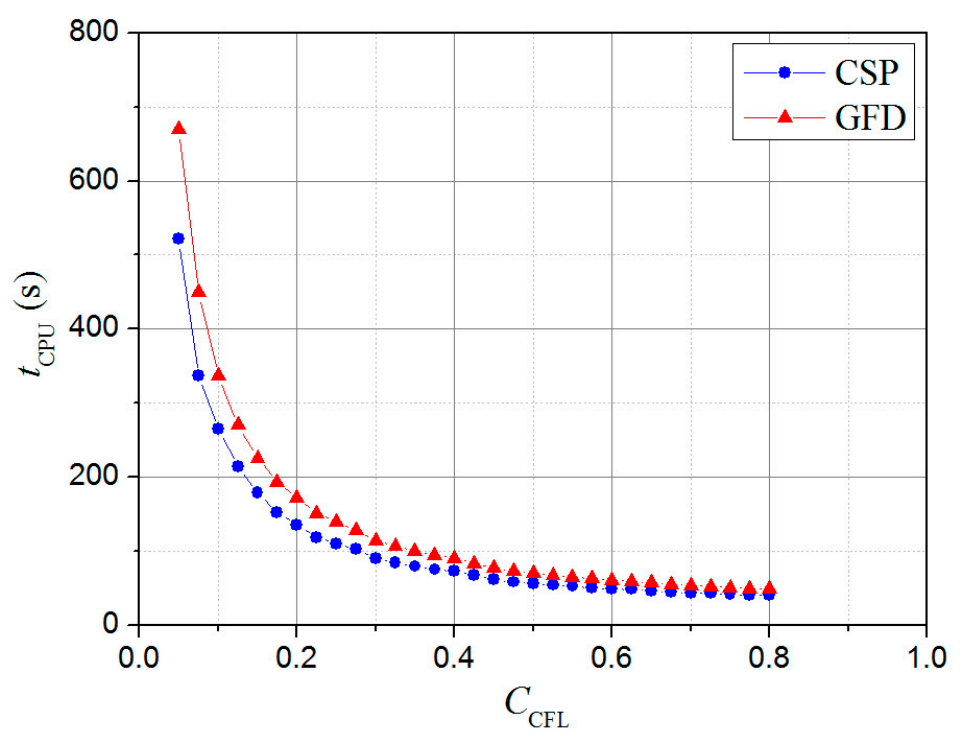

Fig. 26 CPU time curves for CSP and GFD solutions of LLAPE with different CFL numbers.

For further evaluation, Table 4 compares $\varepsilon_{\text {pre }}$ and $t_{\mathrm{CPU}}$ of two meshfree methods in solving LAPE and LLAPE.

Table 4 Comparison of CSP, and GFD methods in solving LAPE and LLAPE with rotational flow.

\begin{tabular}{ccccc}
\hline & \multicolumn{2}{c}{ CSP } & \multicolumn{2}{c}{ GFD } \\
Equations & $\varepsilon_{\text {pre }}$ & $t$ CPU & $\varepsilon_{\text {pre }}$ & $t$ CPU \\
& $\left(\times 10^{-5}\right)$ & $(\mathrm{s})$ & $\left(\times 10^{-5}\right)$ & $(\mathrm{s})$ \\
\hline LAPE & 1.8549 & 223 & 1.4699 & 198 \\
LLAPE & 1.8612 & 136 & 1.4761 & 171 \\
\hline
\end{tabular}

The table reveals that both LAPE and LLAPE are able to solve 2D acoustic problems in rotational background flow. LLAPE can save some computational time with acceptable loss in the accuracy compared with LAPE. Similar to the previous three cases, the GFD method 
shows better accuracy than the CSP method.

\section{Conclusions}

A Lagrangian approach for computational acoustics is presented and implemented numerically, using the SPH, CSP, and GFD methods to solve LAPE and LLAPE. In this Lagrangian approach, both acoustic and fluid variables are supposed to be the physical properties of a fluid particle which can move through both space and time, and LAPE and LLAPE are derived based on the hydrodynamic/acoustic splitting method. Applications to modeling sound propagation in moving fluids are outlined with four examples, and a comparison of the LAPE and LLAPE using the three meshfree methods is presented. Effects of particle spacing, smoothing length, and Courant number on SPH, CSP and GFD methods are evaluated.

The error analysis show that the present Lagrangian approach gives good agreement with exact solutions, and LLAPE can save some computational time with acceptable accuracy loss compared with LAPE. For four test cases, three meshfree methods are accurate for the time domain simulation of acoustic waves. The CSP and GFD method are convergent for all cases considered, while the standard SPH method is not. The GFD method is more accurate, but need a smaller CFL number to keep low numerical error, which increases computational time.

As illustrated in this paper, the Lagrangian approach is supposed to have potential for acoustic problems like bubble acoustics and combustion noise. Nevertheless, before engineering applications, variety of boundary conditions for the present Lagrangian approach still need to be analyzed with more numerical experiments. This issue is still under study and will be reported in a subsequent paper.

\section{Acknowledgments}

This study was supported by the National Natural Science Foundation of China (No. 40976058 and 51379083), and the Independent Innovation Foundation of Huazhong University of Science and Technology (No. 01-18-140019). Y. O. Zhang was supported by the China Scholarship Council (201406160032).

\section{References}

[1] P. Moin, K. Mahesh, Direct numerical simulation: a tool in turbulence research. Annual Review of Fluid Mechanics 30 (1998) 539-578.

[2] C. Bailly, D. Juve, Numerical solution of acoustic propagation problems using 
linearized Euler equations. AIAA journal 38 (2000) 22-29.

[3] M.J. Lighthill, On sound generated aerodynamically. I. General theory. Proceedings of the Royal Society of London. Series A. Mathematical and Physical Sciences 211 (1952) 564-587.

[4] J.E. Ffowcs Williams, D.L. Hawkings, Sound generation by turbulence and surfaces in arbitrary motion. Philosophical Transactions of the Royal Society of London. Series A, Mathematical and Physical Sciences 264 (1969) 321-342.

[5] J.C. Hardin, D.S. Pope, An acoustic/viscous splitting technique for computational aeroacoustics. Theoretical and Computational Fluid Dynamics 6 (1994) 323-340.

[6] W.Z. Shen, J. Nørkær Sørensen, Aeroacoustic modelling of low-speed flows. Theoretical and Computational Fluid Dynamics 13 (1999) 271-289.

[7] O.A. Godin, Reciprocity and energy theorems for waves in a compressible inhomogeneous moving fluid. Wave Motion 25 (1997) 143-167.

[8] L.M. Brekhovskikh, O.A. Godin, Acoustics of Layered Media II: Point Sources and Bounded Beams, Volume 2, Springer, 1999.

[9] J.H. Seo, Y.J. Moon, Perturbed compressible equations for aeroacoustic noise prediction at low mach numbers. AIAA journal 43 (2005) 1716-1724.

[10] J.H. Seo, Y.J. Moon, Linearized perturbed compressible equations for low Mach number aeroacoustics. Journal of Computational Physics 218 (2006) 702-719.

[11] S.Z. Wang, Finite-difference time-domain approach to underwater acoustic scattering problems. The Journal of the Acoustical Society of America 99 (1996) 1924-1931.

[12] I. Harari, A survey of finite element methods for time-harmonic acoustics. Computer Methods in Applied Mechanics and Engineering 195 (2006) 1594-1607.

[13] P.K. Kythe, An Introduction to Boundary Element Methods, Volume 4, CRC Press, 1995.

[14] C. Wang, L. Huang, Time-domain simulation of acoustic wave propagation and interaction with flexible structures using Chebyshev collocation method. Journal of Sound and Vibration 331 (2012) 4343-4358.

[15] Z.C. He, G.Y. Li, E. Li, Z.H. Zhong, G.R. Liu, Mid-frequency acoustic analysis using edge-based smoothed tetrahedron radialpoint interpolation methods. International Journal of Computational Methods 11 (2014) 1350103.

[16] A. Tadeu, P. Stanak, J. Sladek, V. Sladek, Coupled BEM-MLPG acoustic analysis for non-homogeneous media. Engineering Analysis with Boundary Elements 44 (2014) 161169.

[17] T. Leighton, The Acoustic Bubble, Academic Press, 2012.

[18] A. Schwarz, J. Janicka, Combustion Noise. Volume 102, Springer Science \& 
Business Media, 2009.

[19] H.K. Kytömaa, Theory of sound propagation in suspensions: a guide to particle size and concentration characterization. Powder Technology 82 (1995) 115-121.

[20] J.F. Price, Lagrangian and Eulerian representations of fluid flow: Kinematics and the equations of motion, Master Thesis, Woods Hole Oceanographic Institution, Woods Hole, 2006.

[21] M.B. Liu, G.R. Liu, Smoothed particle hydrodynamics (SPH): an overview and recent developments. Archives of Computational Methods in Engineering 17 (2010) 25-76.

[22] M.B. Liu, G.R. Liu, Z. Zong, An overview on smoothed particle hydrodynamics. International Journal of Computational Methods 5 (2008) 135-188.

[23] J.J. Monaghan, Smoothed particle hydrodynamics and its diverse applications. Annual Review of Fluid Mechanics 44 (2012) 323-346.

[24] C.T. Wolfe, S.K. Semwal, Acoustic modeling of reverberation using smoothed particle hydrodynamics, Master Thesis, University of Colorado, 2008.

[25] P. Hahn, D. Negrut, On the use of meshless methods in acoustic simulations. In ASME 2009 International Mechanical Engineering Congress and Exposition, American Society of Mechanical Engineers, 2009, pp. 185-199.

[26] F. Treyssede, G. Gabard, M.B. Tahar, A mixed finite element method for acoustic wave propagation in moving fluids based on an Eulerian-Lagrangian description. The Journal of the Acoustical Society of America 113 (2003) 705-716.

[27] G. Gabard, E. Lefrancois, M.B. Tahar, Aeroacoustic noise source simulations based on Galbrun's equation. AIAA Paper 2892 (2004) 2004.

[28] R. Ewert, W. Schröder, Acoustic perturbation equations based on flow decomposition via source filtering. Journal of Computational Physics 188 (2003) 365-398.

[29] R.A. Uras, C.T. Chang, Y. Chen, W.K. Liu, Multiresolution reproducing kernel particle methods in acoustics. Journal of Computational Acoustics 5 (1997) 71-94.

[30] P. Bouillard, S. Suleaub, Element-Free Galerkin solutions for Helmholtz problems: fomulation and numerical assessment of the pollution effect. Computer Methods in Applied Mechanics and Engineering 162 (1998) 317-335.

[31] C.C. Tsai, D.L. Young, C.L. Chiu, C.M. Fan, Numerical analysis of acoustic modes using the linear least squares method of fundamental solutions. Journal of Sound and Vibration 324 (2009) 1086-1110.

[32] Z. He, P. Li, G. Zhao, H. Chen, A meshless Galerkin least-square method for the Helmholtz equation. Engineering Analysis with Boundary Elements 35 (2011) 868-878.

[33] Z.J. Fu, W. Chen, Y. Gu, Burton-Miller-type singular boundary method for acoustic radiation and scattering. Journal of Sound and Vibration 333 (2014) 3776-3793. 
[34] L.B. Lucy, A numerical approach to the testing of the fission hypothesis. The Astronomical Journal 82 (1977) 1013-1024.

[35] R.A. Gingold, J.J. Monaghan, Smoothed particle hydrodynamics: theory and application to non-spherical stars, Monthly Notices of the Royal Astronomical Society 181 (1977) 375-389.

[36] V. Springel, Smoothed particle hydrodynamics in astrophysics. Annual Review of Astronomy and Astrophysics 48 (2010) 391-430.

[37] G.R. Liu, M.B. Liu, Smoothed Particle Hydrodynamics: A Meshfree Particle Method, World Scientific, 2003.

[38] J.K. Chen, J.E. Beraun, T.C. Carney, A corrective smoothed particle method for boundary value problems in heat conduction, Computer Methods in Applied Mechanics and Engineering 46 (1999) 231-252.

[39] J.K. Chen, J.E. Beraun, C.J. Jih, Completeness of corrective smoothed particle method for linear elastodynamics, Computational Mechanics, 24 (1999) 273-285.

[40] A. Colagrossi, M. Landrini, Numerical simulation of interfacial flows by smoothed particle hydrodynamics. Journal of Computational Physics 191 (2003) 448-475.

[41] J.J. Monaghan, Simulating free surface flows with SPH. Journal of Computational Physics 110 (1994) 399-406.

[42] J.J. Monaghan, Implicit SPH drag and dusty gas dynamics. Journal of Computational Physics 138 (1997) 801-820.

[43] Y.O. Zhang, T. Zhang, H. Ouyang, T.Y. Li, SPH simulation of sound propagation and interference, Proceedings of the 5th International Conference on Computational Method (ICCM'14), Cambridge, July 2014, pp. 1-6.

[44] Y.O. Zhang, T. Zhang, H. Ouyang, T.Y. Li, SPH simulation of acoustic waves: effects of frequency, sound pressure, and particle spacing. Mathematical Problems in Engineering 7 (2015) 348314.

[45] Y.O. Zhang, T. Zhang, H. Ouyang, T.Y. Li, Efficient SPH simulation of timedomain acoustic wave propagation. Engineering Analysis with Boundary Elements 62 (2016) 112-122.

[46] P.S. Jensen, Finite difference techniques for variable grids. Computers and Structures 2 (1972) 17-29.

[47] N. Perrone, R. Kao, A general finite difference method for arbitrary meshes. Computers and Structures 5 (1975) 45-57.

[48] T. Liszka, J. Orkisz, The finite difference method at arbitrary irregular grids and its application in applied mechanics. Computers and Structures 11 (1980) 83-95.

[49] J.J. Benito, F. Urena, L. Gavete, Influence of several factors in the generalized finite 
difference method. Applied Mathematical Modelling 25 (2001) 1039-1053.

[50] L. Gavete, M.L. Gavete, J.J. Benito, Improvements of generalized finite difference method and comparison with other meshless method. Applied Mathematical Modelling 27 (2003) 831-847.

[51] J.J. Benito, F. Urena, L. Gavete, Solving parabolic and hyperbolic equations by the generalized finite difference method. Journal of Computational and Applied Mathematics 209 (2007) 208-233.

[52] J. Wei, S. Wang, Q. Hou, J. Dang, Generalized finite difference time domain method and its application to acoustics. Mathematical Problems in Engineering 7 (2015) 640305.

[53] J.J. Monaghan, J.C. Lattanzio, A refined particle method for astrophysical problems. Astronomy and Astrophysics 149 (1985) 135-143.

[54] M. Kelager, Lagrangian fluid dynamics using smoothed particle hydrodynamics, PhD Thesis, University of Copenhagen, 2006.

[55] J.J. Monaghan, Smoothed particle hydrodynamics. Reports on Progress in Physics 68 (2005): 1703-1759.

[56] L. Gavete, F. Ureña, J.J. Benito, E. Salete, Solving third and fourth order partial differential equations using GFDM: application to solve problems of plates. International Journal of Computer Mathematics 89 (2012) 366-376.

[57] M.D. Dahl, Fourth computational aeroacoustics (CAA) workshop on benchmark problems, NASA/CP-2004-212954, E-14393, 2004.

[58] R. Von Mises, H. Geiringer, G.S.S. Ludford, Mathematical theory of compressible fluid flow, Courier Corporation, 2004.

[59] R. Fatehi, M.T. Manzari, Error estimation in smoothed particle hydrodynamics and a new scheme for second derivatives. Computers and Mathematics with Applications 61 (2011) 482-498.

[60] Q. Zhu, L. Hernquist, Y. Li, Numerical convergence in smoothed particle hydrodynamics. The Astrophysical Journal 800 (2015) 6.

[61] J.C. Hardin, J.R. Ristorcelli, ICASE/LaRC workshop on benchmark problems in computational aeroacoustics. National Aeronautics and Space Administration, Virginia, October 1994.

(C) 2017 by the authors; licensee Preprints, Basel, Switzerland. This article is an open access article distributed under the terms and conditions of the Creative Commons by Attribution (CC-BY) license (http://creativecommons.org/licenses/by/4.0/). 